University of Wollongong

Research Online

Faculty of Engineering and Information

Faculty of Engineering and Information

Sciences - Papers: Part A

Sciences

$1-1-2015$

\title{
Application of the largest Lyapunov exponent algorithm for feature extraction in low speed slew bearing condition monitoring
}

Wahyu Caesarendra

University of Wollongong,wc026@uowmail.edu.au

Prabuono Buyung Kosasih

University of Wollongong, buyung@uow.edu.au

A Kiet Tieu

University of Wollongong, ktieu@uow.edu.au

Craig A. S Moodie

University of Wollongong, cam920@uow.edu.au

Follow this and additional works at: https://ro.uow.edu.au/eispapers

Part of the Engineering Commons, and the Science and Technology Studies Commons

Research Online is the open access institutional repository for the University of Wollongong. For further information contact the UOW Library: research-pubs@uow.edu.au 


\title{
Application of the largest Lyapunov exponent algorithm for feature extraction in low speed slew bearing condition monitoring
}

\author{
Abstract \\ This paper presents a new application of the largest Lyapunov exponent (LLE) algorithm for feature \\ extraction method in low speed slew bearing condition monitoring. The LLE algorithm is employed to \\ measure the degree of non-linearity of the vibration signal which is not easily monitored by existing \\ methods. The method is able to detect changes in the condition of the bearing and demonstrates better \\ tracking of the progressive deterioration of the bearing during the 139 measurement days than \\ comparable methods such as the time domain feature methods based on root mean square (RMS), \\ skewness and kurtosis extraction from the raw vibration signal and also better than extracting similar \\ features from selected intrinsic mode functions (IMFs) of the empirical mode decomposition (EMD) \\ result. The application of the method is demonstrated with laboratory slew bearing vibration data and \\ industrial bearing data from a coal bridge reclaimer used in a local steel mill.

\section{Keywords} \\ speed, low, extraction, feature, algorithm, condition, exponent, monitoring, lyapunov, largest, application, \\ slew, bearing

\section{Disciplines} \\ Engineering | Science and Technology Studies

\section{Publication Details} \\ Caesarendra, W., Kosasih, B., Tieu, A. Kiet . \& Moodie, C. A. S. (2015). Application of the largest Lyapunov \\ exponent algorithm for feature extraction in low speed slew bearing condition monitoring. Mechanical \\ Systems and Signal Processing, 50-51 116-138.
}

This journal article is available at Research Online: https://ro.uow.edu.au/eispapers/2905 
Manuscript for Mechanical Systems and Signal Processing

\title{
Application of the largest Lyapunov exponent algorithm for feature extraction in low speed slew bearing condition monitoring
}

\author{
Wahyu Caesarendra*a, $^{\mathrm{a}}$, Buyung Kosasih ${ }^{\mathrm{a}}$, Anh Kiet Tieu ${ }^{\mathrm{a}}$, Craig A.S. Moodie ${ }^{\mathrm{a}}$ \\ ${ }^{a}$ School of Mechanical, Materials and Mechatronic Engineering, University of Wollongong, Wollongong, New South \\ Wales 2522, Australia \\ ${ }^{b}$ Mechanical Engineering Department, Diponegoro University, Tembalang, Semarang 50275, Indonesia
}

\section{* Corresponding Author:}

Wahyu Caesarendra

School of Mechanical, Materials and Mechatronic Engineering,

University of Wollongong,

Wollongong, NSW 2522, Australia

Phone: $612+42213555$

Fax: $612+42213477$

E-mail:wc026@uowmail.edu.au

Total number of pages: 35

Total number of figures: 16

Total number of tables: 9 


\title{
Application of the largest Lyapunov exponent algorithm for feature extraction in low speed slew bearing condition monitoring
}

\begin{abstract}
This paper presents a new application of the largest Lyapunov exponent (LLE) algorithm for feature extraction method in low speed slew bearing condition monitoring. The LLE algorithm is employed to measure the degree of non-linearity of the vibration signal which is not easily monitored by existing methods. The method is able to detect changes in the condition of the bearing and demonstrates better tracking of the progressive deterioration of the bearing during the 139 measurement days than comparable methods such as the time domain feature methods based on root mean square (RMS), skewness and kurtosis extraction from the raw vibration signal and also better than extracting similar features from selected intrinsic mode functions (IMFs) of the empirical mode decomposition (EMD) result. The application of the method is demonstrated with laboratory slew bearing vibration data and industrial bearing data from a coal bridge reclaimer used in a local steel mill.
\end{abstract}

Keywords: Empirical mode decomposition; Feature extraction; Largest Lyapunov exponent; Low speed slew bearing.

\section{Nomenclature}

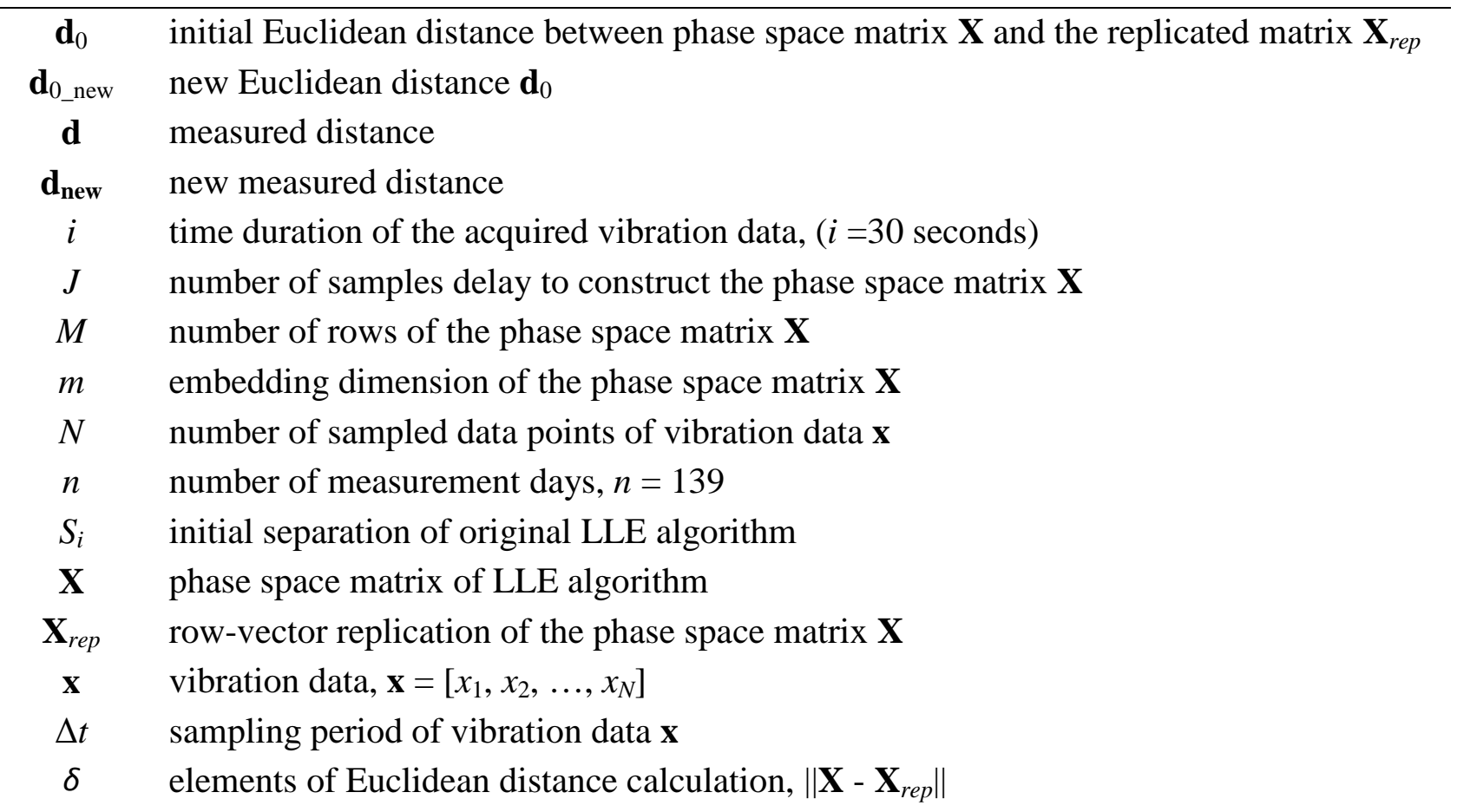


$\lambda_{1} \quad$ largest Lyapunov exponent (LLE)

$\mu \quad$ number of nearest neighbors after and before the zero value

$\Delta_{\mathrm{a}} \quad$ minimum value of $\mathbf{d}_{0 \_ \text {new }}$

$\Delta_{\mathrm{b}} \quad$ identifier of the minimum value $\Delta_{\mathrm{a}}$

\section{Introduction}

Slew bearings are commonly used in large industrial machineries such as turntables, steel mill cranes, off-shore cranes, rotatable trolleys, excavators, reclaimers, stackers, swing shovels and ladle cars. They typically support high axial and radial loads. In the steel industry, slew bearings are often critical production parts. An unplanned downtime caused by the breakdown of the bearing can be very costly due to the interruption in production. In addition, since it takes a long time to replace the slew bearing due to long manufacturing and delivery times, plants often need to carry spare bearings in order to guard against such unforeseen circumstances, adding extra cost. In order to prevent extended unplanned downtime, an accurate method to monitor and analyse the condition of the bearings is needed.

To date, the signal processing technique based on vibration is the main technique used in the detection of change of bearing conditions and as a warning of impending deterioration of rotating machinery. In the case of slew bearings, special signal processing is necessary to detect the changes in the condition of the bearing based on the vibration signal. With appropriate signal processing, the fault signal can be detected from the processed signal. It is well known that the vibration characteristic of rotating machinery is altered when a bearing has failed. As the change is identified by the non-linear trend, a non-linear dynamical analysis technique is necessary for fault detection and diagnosis [1]. Unlike linear dynamic systems where the system has constant natural frequencies and vibrates at the frequency of an externally applied harmonic excitation, in non-linear systems there are amplitude-dependant natural frequencies called 'internal resonances' and this kind of system may vibrate at a frequency different from an externally applied harmonic excitation [2]. In practice, a rolling element bearing has internal resonances due to the different parts of the system (e.g. interactions between shaft and bearing, between rolling element and raceway) vibrating at different frequencies, all with steady amplitudes. The combination of these internal resonances appears in the non-linear vibration signal. Certain methods which can analyze the non-linear time series characteristic (in this paper referred to as the 'vibration signal characteristic') are phase space dissimilarity techniques [3, 4] which are Kolmogorov entropy, fractal dimension, correlation dimension, approximate entropy, permutation entropy, and the largest Lyapunov exponent. These 
techniques use reconstruction vectors or phase space as the input matrix to identify the existence and to measure the degree of non-linearity of the time series data. Most of these techniques have already been applied to vibration signal monitoring for machine health diagnostic, for example: the Kolmogorov-Smirnov test [5], fractal dimension [6], correlation dimension [7], approximate entropy [8] and permutation entropy [9].

This paper presents a new application of the largest Lyapunov exponent (LLE) algorithm as a feature extraction method for low speed slew bearing vibration signals. Although the LLE algorithm has been widely used in some disciplines such as the bio-medical [10,11] and finance [12] areas, it has not been explored previously in vibration-based condition monitoring. For instance, in biomedical applications [10,11], the LLE algorithm is used to analyze the EEG signals to detect epileptic seizures. In finance, [12] the LLE algorithm is used to analyze the stability of electricity prices. The LLE algorithm is usually used for non-linear time series analysis to quantify the appearance of signal non-linearity. Basically, the LLE algorithm measures the exponential divergence (positive or negative) of two initial neighboring trajectories in the phase space based on the Euclidean distance. The LLE algorithm is presented in detail in section 2.

The paper is organized as follows: Section 2 discusses the theoretical background of the LLE algorithm as a non-linear feature extraction method; Section 3 describes the experimental set-up of the laboratory slew bearing test rig; Section 4 describes the coal bridge reclaimer data; Section 5 presents the results and discussion of the vibration-based FFT, statistical time domain feature extraction, empirical mode decomposition (EMD)-based feature extraction and the LLE results including the comparison between the LLE feature and the time domain features extracted from raw vibration data and from selected IMFs of EMD; Section 6 presents results and discussion of coal bridge reclaimer data; Section 7 presents the assessment or evaluation criteria; and Section 8 presents the conclusions of the current work.

\section{The largest Lyapunov exponent (LLE) algorithm}

To analyze the non-linearity or chaotic characteristic of time-series data, the vibration data, $\mathbf{x}=\left(x_{1}, x_{2}, \ldots, x_{N}\right)$ with $N$ samples is reconstructed first. The reconstructed vector is done in phase space [13]. The phase space reconstruction technique used is the method of delays (MOD) introduced by Takens [14]. The phase space has been used in non-linear time series analyses [3-9] where the phase space matrix is defined as follows: 


$$
\mathbf{X}=\left[\begin{array}{rrrrr}
x_{1} & x_{1+J} & x_{1+2 J} & \ldots & x_{1+(m-1) J} \\
x_{2} & x_{2+J} & x_{2+2 J} & \ldots & x_{2+(m-1) J} \\
x_{3} & x_{3+J} & x_{3+2 J} & \ldots & x_{3+(m-1) J} \\
\cdot & \cdot & \cdot & & \cdot \\
\cdot & \cdot & \cdot & & \cdot \\
\cdot & \cdot & \cdot & & \cdot \\
x_{M} & x_{M+J} & x_{M+2 J} & \ldots & x_{M+(m-1) J}
\end{array}\right]
$$

Once $J$ and $m$ are determined, and the sampling rates of the vibration data $N$, is known, the relation between these variables and the number of reconstructed vectors $M$ can be calculated in the following way: $M=N-(m-1) J$. The preparatory LLE algorithm flowchart including the phase space and the initial Euclidean distance calculation is presented in Fig. 1.

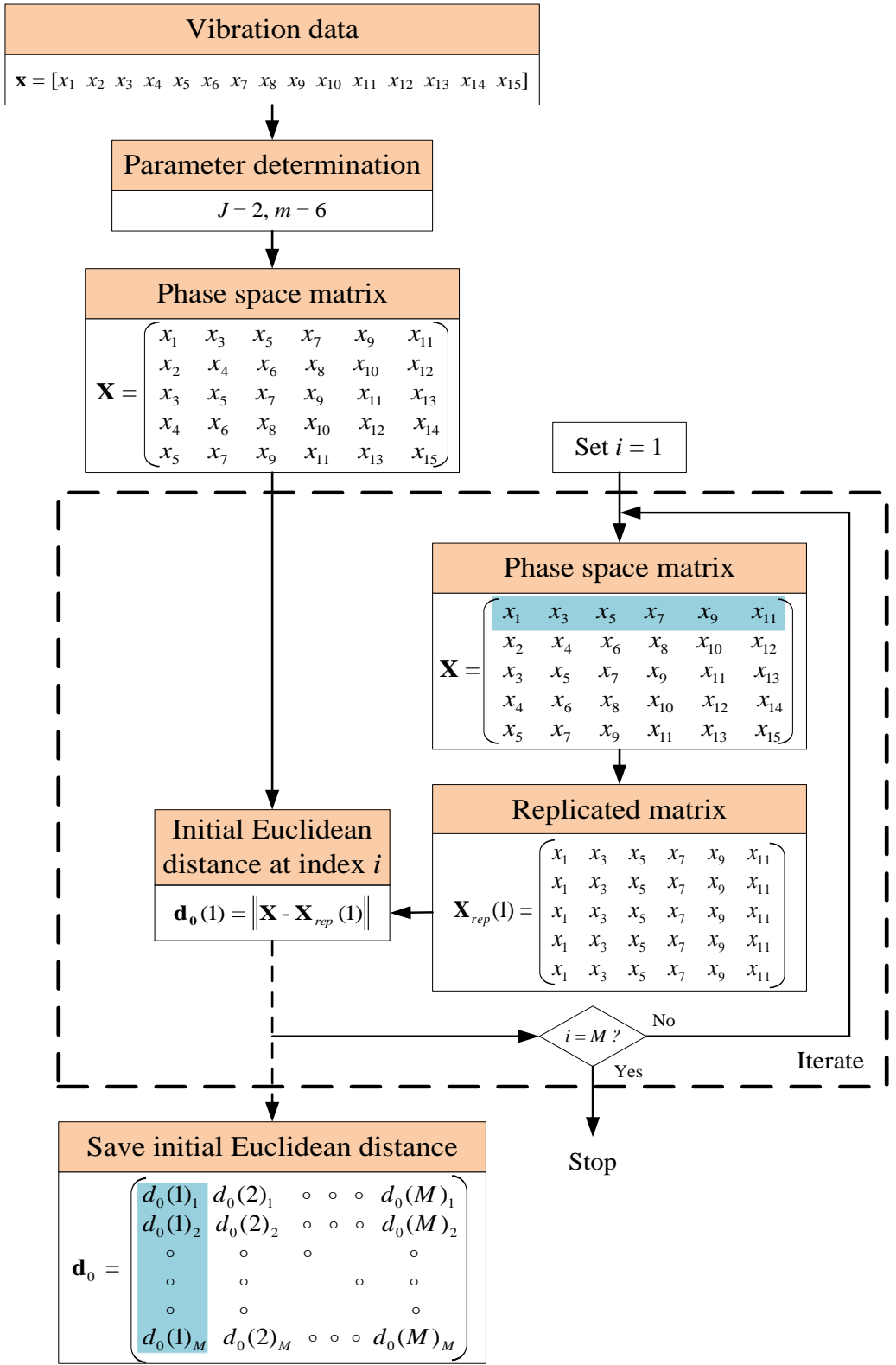

Fig. 1. The flowchart of preparatory LLE algorithm (note: $N=15, J=2$ and $m=6$ ). 
Further explanation of the LLE algorithm in this section is based on the actual slew bearing data. In the case of slew bearing data, the parameters $J$ and $m$ have been determined as $J=48$ and $m=$ 100. The number of samples of slew bearing data $N$ is 4880 . Hence, the number of reconstructed vector $M$ can be calculated, $M=128$. The reason why these values were selected is explained in detail in section 5.4. Once the phase matrix $\mathbf{X}$ and the replicated matrix $\mathbf{X}_{\text {rep }}$ have been obtained, the initial Euclidean distance, vector $\mathbf{d}_{0}$, can be calculated as shown in Fig. 1 . It should be noted that the calculation of the Euclidean distance vector in the LLE algorithm plays an important role. The divergence of the LLE result $\left(\lambda_{1}\right)$ can be analysed based on the Euclidean distance. The divergence represents the characteristic of the vibration signal being analysed. If the bearing vibration signal is linear, typical of normal condition, the calculated Euclidean distance between each row in the phase space matrix is constant and divergence is not detected, and thus the $\lambda_{1}$ will be negative. On the other hand, if the vibration signal has non-linear characteristics, the Euclidean distance between each row in the phase space matrix is no longer constant (it typically manifests exponentially), and thus the value of $\lambda_{1}$ will be positive. The physical reason for the LLE result $\left(\lambda_{1}\right)$ mentioned above is discussed again in detail at the end of the LLE algorithm procedure in this section. However, the important Euclidean distance vector for the LLE result $\left(\lambda_{1}\right)$ is not $\mathbf{d}_{0}$. To get the appropriate Euclidean distance vector for calculating $\lambda_{1}$, the initial Euclidean distance vector $\mathbf{d}_{0}$ described above needs to be processed by the two following steps:

\section{Step 1: Calculate the new initial Euclidean distance value $\mathbf{d}_{0 \_ \text {new }}$}

The first step to process the Euclidean distance vector $\mathbf{d}_{0}(i)$ is to determine the identifier of the minimum value of the Euclidean distance vector $\mathbf{d}_{0}(i)$ for each index $i$. The index $i$ in the case of slew bearings is $i=1,2, \ldots, 128$. Prior to the calculation of the minimum identifier, it is necessary to calculate the minimum value of $\mathbf{d}_{0}(i)$ for each $i$, then the identifier of the minimum value of $\mathbf{d}_{0}(i)$ for each $i$ can be obtained. If we compute the minimum value directly from $\mathbf{d}_{0}(i)$ as $i$ progresses from $1,2, \ldots, 128$, the minimum value will always be 'zero'. The reason for the 'zero' value is that at the particular index $i$, for instance at $i=1$, the $\mathbf{d}_{0}(1)$ is computed from $\left\|\mathbf{X}-\mathbf{X}_{\text {rep }}(1)\right\|$. As mentioned before, $\mathbf{X}_{\text {rep }}(1)$ is obtained by replicating the $1^{\text {st }}$ row of the phase space matrix $\mathbf{X}$. If the Euclidean norm between $\mathbf{X}$ and $\mathbf{X}_{\text {rep }}(1)$ is calculated, the first row of the Euclidean distance vector $\mathbf{d}_{0}$ will be zero value, because the first row element of the phase space matrix $\mathbf{X}$ and the replicated matrix $\mathbf{X}_{r e p}$ are identical. When $i$ progresses up to, for example, $i=64$, the $\mathbf{d}_{0}(64)$ is computed from $\| \mathbf{X}$ $\mathbf{X}_{r e p}(64) \|$. In a similar way, the matrix $\mathbf{X}_{r e p}(64)$ is obtained by replicating the $64^{\text {th }}$ row of the phase space matrix $\mathbf{X}$. If the Euclidean norm between $\mathbf{X}$ and $\mathbf{X}_{\text {rep }}(64)$ is applied, the $64^{\text {th }}$ row of the Euclidean distance vector $\mathbf{d}_{0}$ will be zero as well. The process which produces the zero value of $\mathbf{d}_{0}$ 
as $i$ progresses is repeated up to the last $i$ index, that is $i=128$. The illustration of the three calculation examples of $\mathbf{d}_{0}$ at three different $i$ (at $i=1$, at $i=64$ and at $i=128$ ) are shown in Figs. 2(a)-4(a).

To overcome the problem mentioned above, an additional constraint is employed in the LLE algorithm to remove the 'zero' value as $i$ progresses and to change nearest zero value neighbors become constant (to provide a clearer illustration figure, a maximum value of $\mathbf{d}_{0}$ is used as a constant value). The reason why the nearest zero value neighbors need to be changed to a constant is because the nearest zero value neighbors of $\mathbf{d}_{0}$ shows some transient behavior as shown in Figs. 2(a)-4(a). This is important to ensure an accurate measurement of the new initial distance value $\mathbf{d}_{0 \_ \text {new }}$. Once the transient part of $\mathbf{d}_{0}$ has been removed, the minimum value of the Euclidean distance vector $\mathbf{d}_{0}(i)$ will be calculated from the stationary part. To determine the constraint mentioned above, another index called $j$ is used inside the sub-routine of index $i$ in the LLE algorithm. The constraint is defined as follows:

$$
|i-j| \leq \mu
$$

where $j=1,2, \ldots, M$ and $\mu$ is the number of nearest zero value neighbors that need to be changed to a constant value. The $\mu$ in this paper is selected as 50 (the reason is given in Section 5.4). In addition, Eq. (2) means: if the absolute value of subtraction $i^{\text {th }}$ and $j^{\text {th }}$ as $i$ and $j$ progresses from 1 to 128 is less than or equal to the $\mu$, the element of $\mathbf{d}_{0}$ (with respect to current $j$ ) is replaced by the highest value of $\mathbf{d}_{0}(i)$. A more detail explanation of Eq. (2) is presented in the LLE algorithm in Table 1. The new Euclidean distance vector after the LLE algorithm is shown in Table 1 and is called the new Euclidean distance vectors $\mathbf{d}_{0 \_n e w}(j)$. It can be seen from Table 1 that the algorithm consists of two sub-routines based on the indices $i$ and $j$. The results of $\mathbf{d}_{0 \_n e w}$ are presented in Figs. 2(b)-4(b). It can be seen in Fig. 2(b) that when $i=1$, the fifty data points after the nearest neighbors of the zero value are replaced by the maximum value and became constant. Further example $i=64$, when $i=64$, the nearest neighbors which need to be changed are 50 points before the zero value and 50 points after the zero value. Thus the result is shown in Fig. 3(b). When $i$ reaches the last number i.e. $i=128$, the fifty points before the nearest neighbors of the zero value are replaced by the maximum value and became constant as well, as shown in Fig. 4(b). Note that points are the result of Eq. (2) or line 6 in the LLE algorithm for $j$ 's progression from 1, 2, .,, 128.

Once the $\mathbf{d}_{0 \_ \text {new }}(j)$ is computed, the minimum value of $\mathbf{d}_{0 \_ \text {new }}(j)$ denoted by $\Delta_{a}(i)$ and the index of the minimum value as denoted by $\Delta_{b}(i)$ are defined as follows:

$$
\left[\Delta_{a}(i), \Delta_{b}(i)\right]=\min \left(\mathbf{d}_{0 \_ \text {new }}(j)\right)
$$


Eq. (3) is incorporated in the LLE algorithm shown in Table 1 line 11 inside the sub-routine of index $i$. It should be noted that the minimum value $\Delta_{\mathrm{a}}(i)$ is no longer used for further calculations. Only the identifier of the minimum distance value $\Delta_{b}(i)$ is used again in the next step. This index is necessary to create a new matrix, matrix $\mathbf{X}\left(\Delta_{b}(i)\right)$. The element of the matrix $\mathbf{X}\left(\Delta_{b}(i)\right)$ at particular index $i$ is based on the result of $\Delta_{b}(i)$. Further use of matrix $\mathbf{X}\left(\Delta_{b}(i)\right)$ is shown later in Eq. (4) and Table 2 line 19.

Table 1. The LLE algorithm showing the subroutines of $i$ and $j$ in order to calculate the new Euclidean distance $\mathbf{d}_{0 \_ \text {new. }}$

\section{LLE Algorithm}

1: $\quad \mathbf{X}, \mathbf{X}_{\text {rep }}$ and $\mathbf{d}_{0}$ (are calculated from Fig. 1)

2: $\quad \mu$ is predetermined mean period (e.g. $\mu=50)$

3: Create the new matrix $\mathbf{d}_{0 \_n e w}$. Set $\mathbf{d}_{0 \_n e w}=\mathbf{d}_{0}$ initially

4: $\quad$ for $i=1$ to $M$ do

5: $\quad$ for $j=1$ to $M$ do

6: $\quad$ if $|j-i| \leq \mu$ do

7: $\quad \mathbf{d}_{0 \_ \text {new }}(j)=\max \left(\mathbf{d}_{0}\right)$

8: $\quad$ end if

9: $\quad$ end for $j$

10: $\quad$ Vector $\mathbf{d}_{0 \_ \text {new }}$ obtained. This vector contain $1,2, \ldots M$ row

11: $\quad$ Find the minimum value of $\mathbf{d}_{0 \_ \text {new }}(j)$ and its identifier: $\left[\Delta_{\mathrm{a}}(i), \Delta_{\mathrm{b}}(i)\right]=\min \left(\mathbf{d}_{0 \_ \text {new }}(j)\right)$

12: $\quad$ end for $i$

a

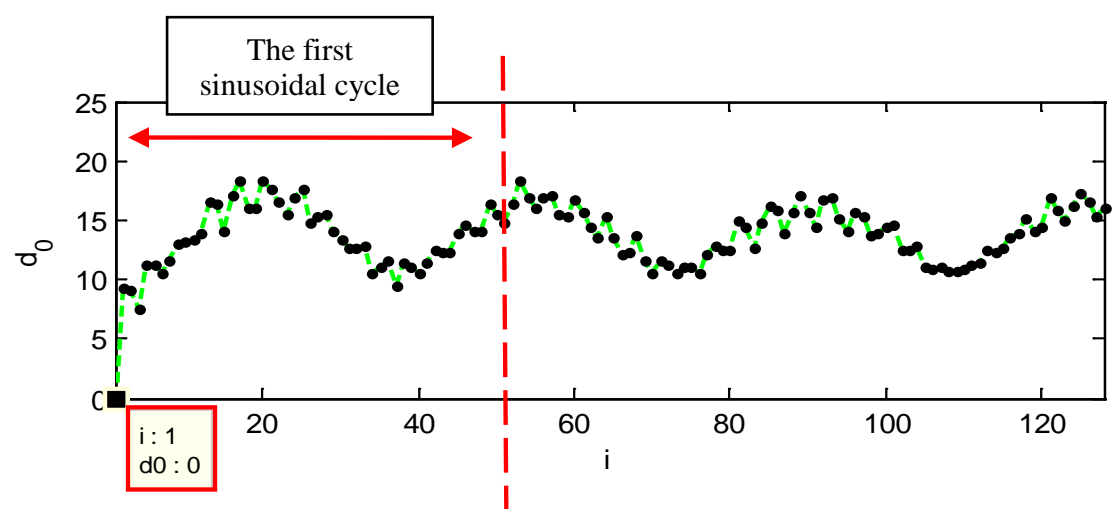

b

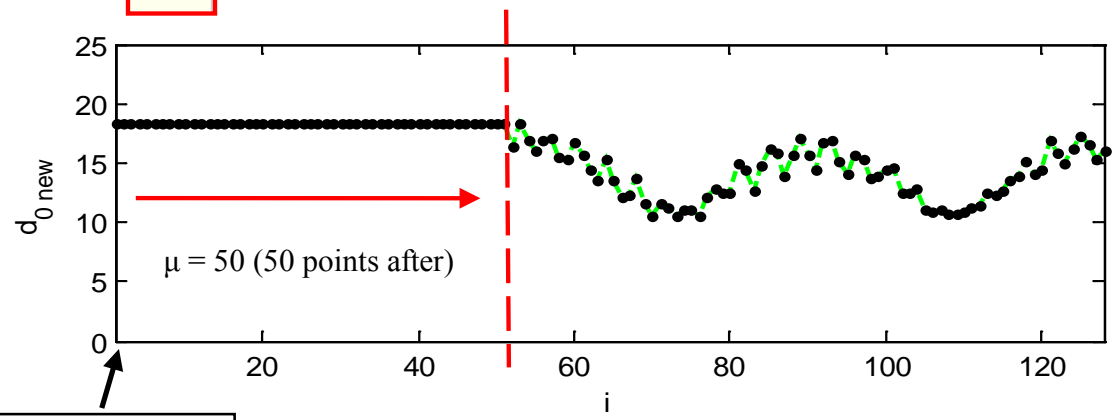

Zero value point 
Fig. 2. (a) The initial Euclidean distance $\mathbf{d}_{0}$ at $i=1$; (b) The new initial Euclidean distance value $\mathbf{d}_{0 \_ \text {new }}$ at $i=1$.

a

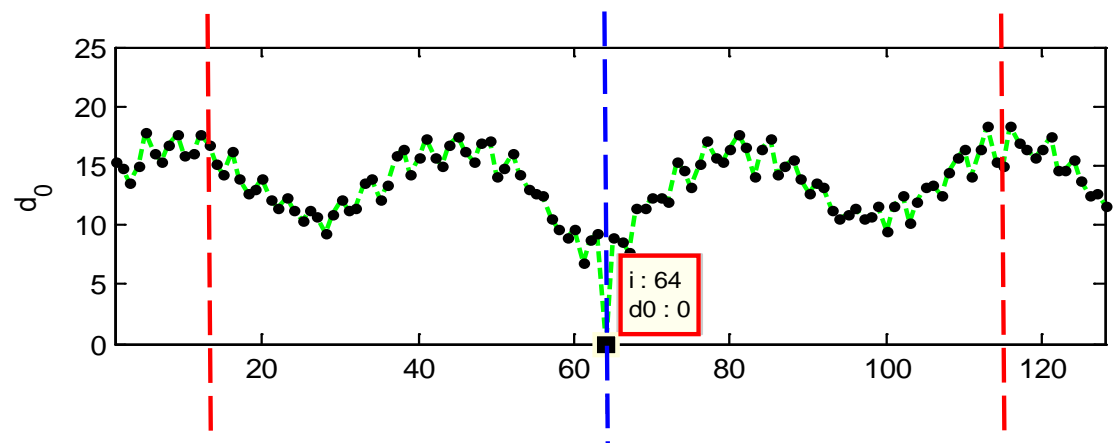

b

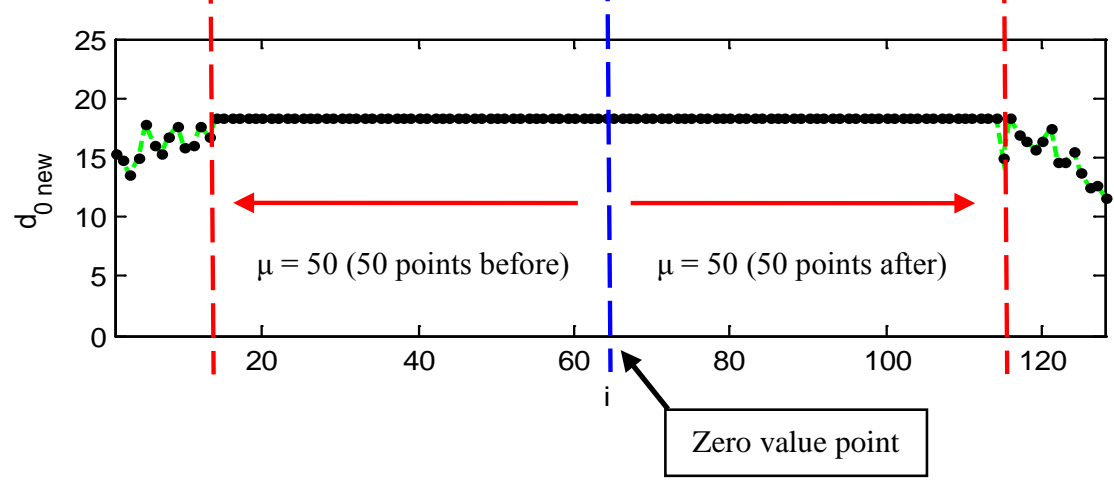

Fig. 3. (a) The initial Euclidean distance $\mathbf{d}_{0}$ at $i=64$; (b) The new initial Euclidean distance value $\mathbf{d}_{0 \_ \text {new }}$ at $i=64$.

a

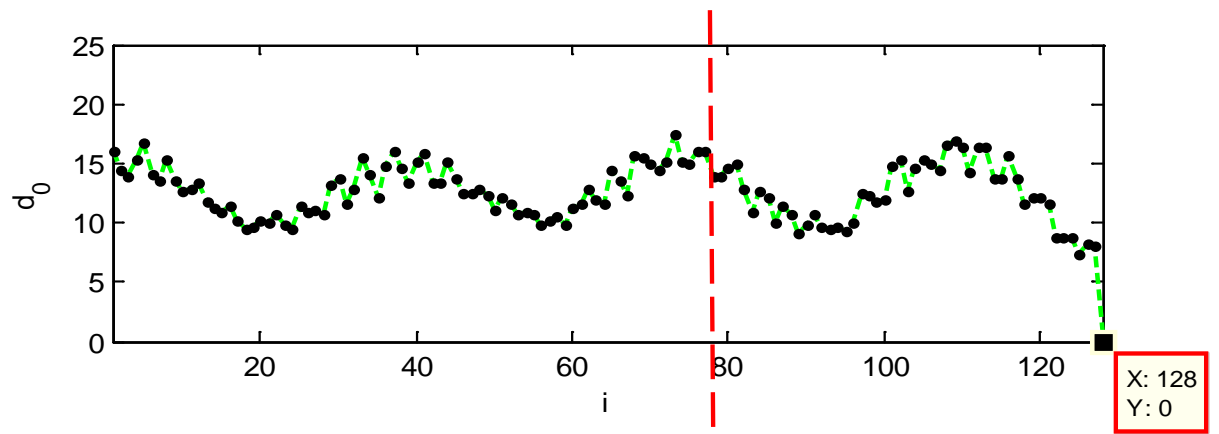

b

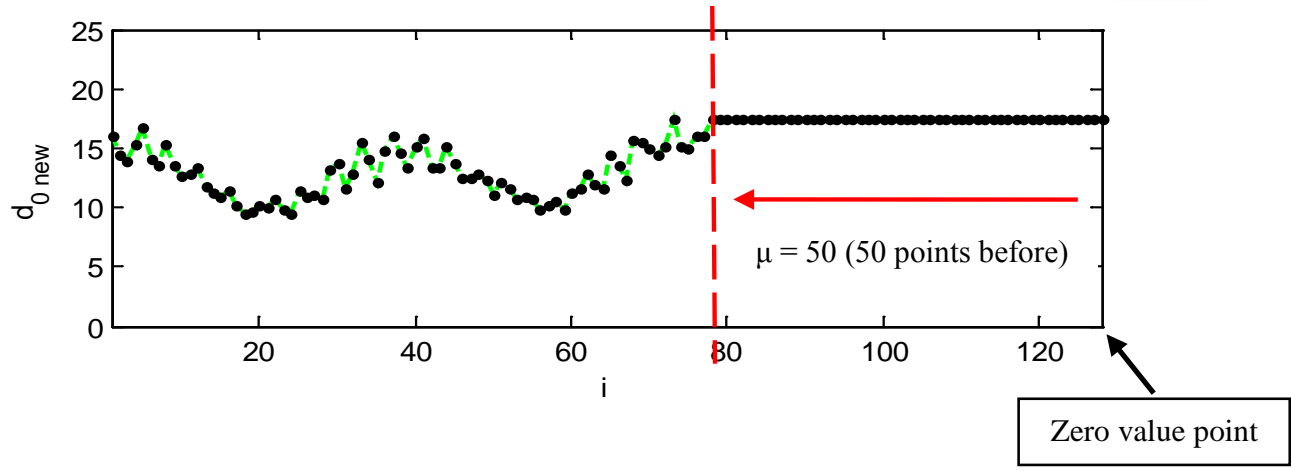

Fig. 4. (a) The initial Euclidean distance $\mathbf{d}_{0}$ at $i=128$; (b) The new initial Euclidean distance value $\mathbf{d}_{0 \_ \text {new }}$ at $i=128$. 
Step 2: Calculate measured distance $\mathbf{d}$ and new measured distance $\mathbf{d}_{\mathbf{n e w}}$

The next step is the calculation of measured distance $\mathbf{d}(i)$ by estimating the distance between the phase space $\mathbf{X}(i)$ and the new matrix $\mathbf{X}\left(\Delta_{b}(i)\right)$ (with respect to $\left.i\right)$. The elements of matrix $\mathbf{X}\left(\Delta_{b}(i)\right.$ ) is the row vector data set of the phase space $\mathbf{X}$ based on the minimum index $\Delta_{\mathrm{b}}(i)$.

$$
\mathbf{d}(i)=\| \mathbf{X}-\mathbf{X}\left(\Delta_{\mathrm{b}}(i) \|\right.
$$

Even though the LLE result $\left(\lambda_{1}\right)$ can be computed using the measured distance $\mathbf{d}(i)$, to improve the accuracy of the measured distance $\mathbf{d}(i)$, Sato et. al. [16] used $k$ iteration. In this paper $k=1,2, \ldots$, 70 iteration is used. In order to show an adequate exponential graph of the measured distance $\mathbf{d}$ and to decrease the computational time, it is recommended that $k$ be any value which is greater than the mean period $\mu$ and less than the embedding dimension $m$. The algorithm used to improve the accuracy of the measured distance $\mathbf{d}(i)$ is presented in Table 2. The algorithm in Table 2 is an extension of the algorithm shown in Table 1 . The result after the $k$ iteration is called the new measured distance matrix $\mathbf{d}_{\text {new }}(i)$.

Table 2. The algorithm to calculate $\mathbf{d}_{\text {new }}(i)$ based on $k$ iteration and the index of minimum value $\Delta_{\mathrm{b}}(i)$.

\section{LLE Algorithm (Cont.)}

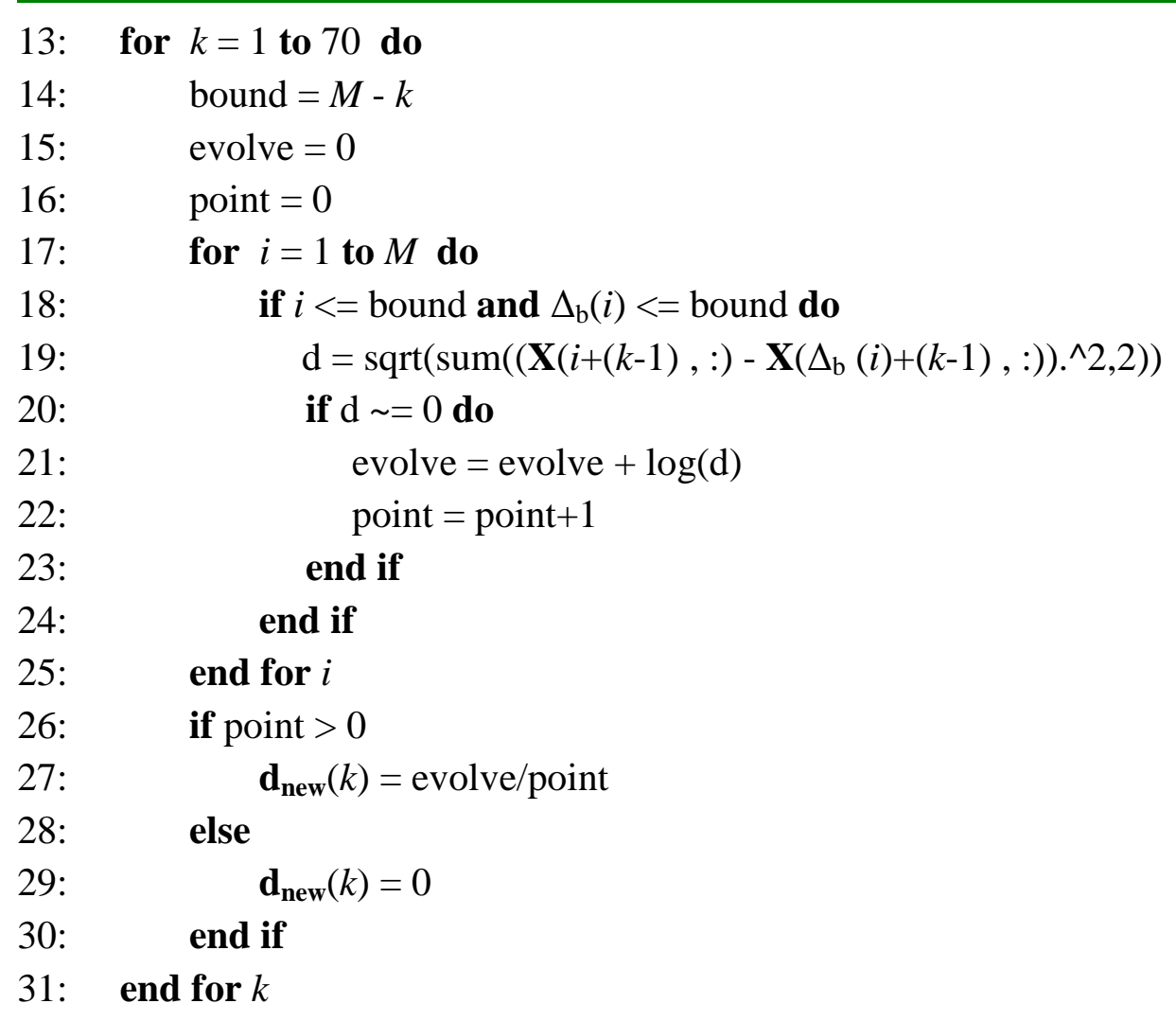


The final step is the calculation of the LLE result $\left(\lambda_{1}\right)$. Using the original largest Lyapunov exponent formula [15], the relation between $\mathbf{d}_{\text {new }}(i)$ and the LLE result $\lambda_{1}(i)$ is defined by the following:

$$
\mathbf{d}_{\text {new }}(i)=S_{i} e^{\lambda_{1}(i . \Delta t)}
$$

By taking the natural logarithm of both sides and removes the part ' $\ln S_{i}$ ', the largest Lyapunov exponent can be computed using a least-square fit equation defined by

$$
\lambda_{1}(i)=\frac{1}{\Delta t} \ln \mathbf{d}_{\text {new }}(i)
$$

where $\lambda_{1}<0$ indicates normal condition and $\lambda_{1}>0$ indicates non-linear condition. The physical interpretation of negative LLE $\left(\lambda_{1}<0\right)$ and positive LLE $\left(\lambda_{1}>0\right)$ is shown in Section 5.4 Figs. 12(a) and (b).

\section{Laboratory slew bearing data}

The vibration of accelerated wear test data used in this paper was acquired from a laboratory slew bearing test rig as shown in Fig. 5 . The test rig was operated in continuous rotation at $1 \mathrm{rpm}$. The slew bearing used was an INA YRT260 type axial/radial bearing supplied by Schaeffler with an inner and outer diameter of $260 \mathrm{~mm}$ and $385 \mathrm{~mm}$, respectively. The vibration data was acquired from four accelerometers installed on the inner radial surface at 90 degrees to each other (see Fig. 5(b)) with $4880 \mathrm{~Hz}$ sampling rates. The accelerometers were IMI608 A11 ICP type sensors. The accelerometers were connected to a high speed Pico scope DAQ (PS3424). The bearing began running in 2006, however in order to provide continuous monitoring and produce run-to-failure bearing data, the bearing data was collected from February to August 2007 (139 days). In order to accelerate the bearing service life, coal dust was injected into the bearing in mid-April 2007 (58 days from the beginning). In practice, especially in steel making companies, the slew bearing is located in the open air where the bearing is exposed to a dusty environment and for this reason, the coal dust was inserted in mid-April to simulate the real working conditions.

The schematic of the slew bearing test rig showing the main drive reducer, the hydraulic load and how the bearing is attached is shown in Fig. 5 (a). The axial load was applied via a hydraulic load of approximately 30 tons. 


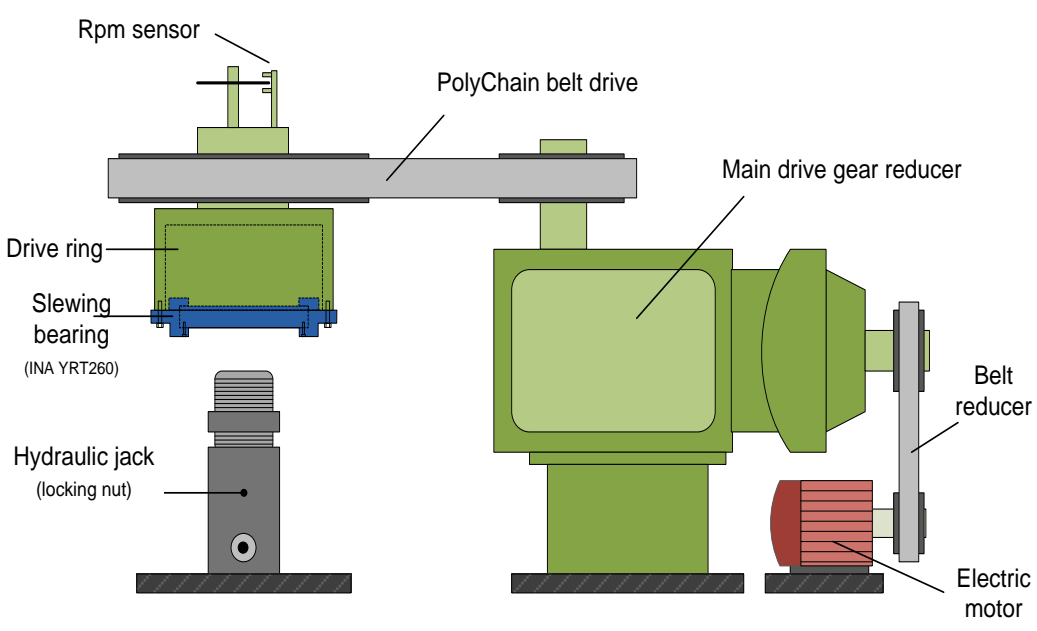

(a)

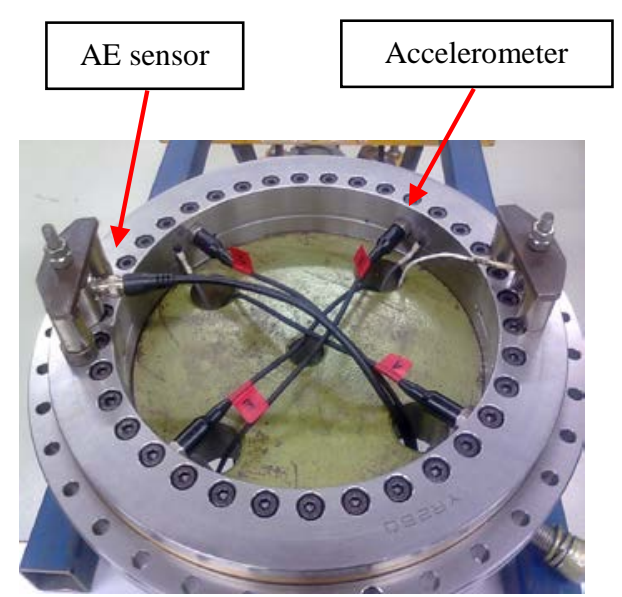

(b)

Fig. 5. (a) Schematic of laboratory slew bearing test-rig; (b) sensor attachment.

\section{Coal bridge reclaimer data}

Industrial bearing data used in this paper was acquired from a slew bearing used in a coal bridge reclaimer. The bearing usually rotates at approximately 4.5rpm. The type of slew bearing used is Rothe Erde 4.3m diameter. The accelerometers employed were IMI512, 500mV/g ICP type piezoelectric. Data was collected from 2003 to 2006 at each sample point, approximately once per month and was captured via an industrialized portable DAQ unit (NI 5102) with a sampling rate of 240.

\section{Results and discussion of laboratory slew bearing data}

\subsection{Vibration-based FFT}

Initially, the vibration-based fast Fourier transform (FFT) was used to identify the dominant frequencies of the vibration signal. The bearing fault frequencies of the slew bearing when the bearing runs at $1 \mathrm{rpm}$ are presented in Table 2. It has been known that if the FFT of the vibration signal contains one or more dominant frequencies which are identical to or match one of the fault frequencies shown in Table 2, a certain fault type has occurred. The three selections of slew bearing data and their FFT are presented in Figs. 6 to 8.

Table 2. Fault frequencies of slew bearing run at $1 \mathrm{rpm}$ [calculation is given in Appendix A].

\begin{tabular}{llc}
\hline \multirow{2}{*}{\multicolumn{1}{c}{ Defect mode }} & \multicolumn{2}{c}{ Fault frequencies $(\mathrm{Hz})$} \\
\cline { 2 - 3 } & Axial & Radial \\
\hline Outer ring (BPFO) & 1.32 & 0.55 \\
Inner ring (BPFI) & 1.37 & 0.55 \\
Rolling element (BSF) & 0.43 & 0.54 \\
\hline
\end{tabular}


The vibration data and its FFT before dust contamination on February 24, one month after dust insertion on May 3 and three days before bearing failure on August 30 are depicted in Fig. 6, Fig. 7 and Fig. 8, respectively. The FFT result in Fig. 6(b) shows that the frequency is less than $100 \mathrm{~Hz}$. After dust insertion, the frequency of the vibration signal is dominated by high frequencies of 1356 $\mathrm{Hz}, 1770 \mathrm{~Hz}$ and $2167 \mathrm{~Hz}$, as shown in Fig. 7(b). When the level of bearing deterioration has increased and the time remaining is close to bearing collapse, the frequency is dominated by a frequency of $103.1 \mathrm{~Hz}$ as shown in Fig. 8(b).

a

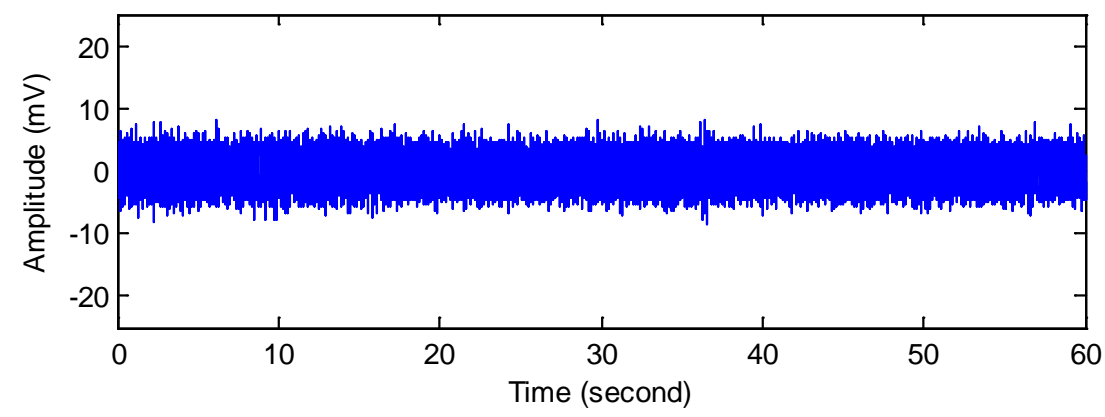

b

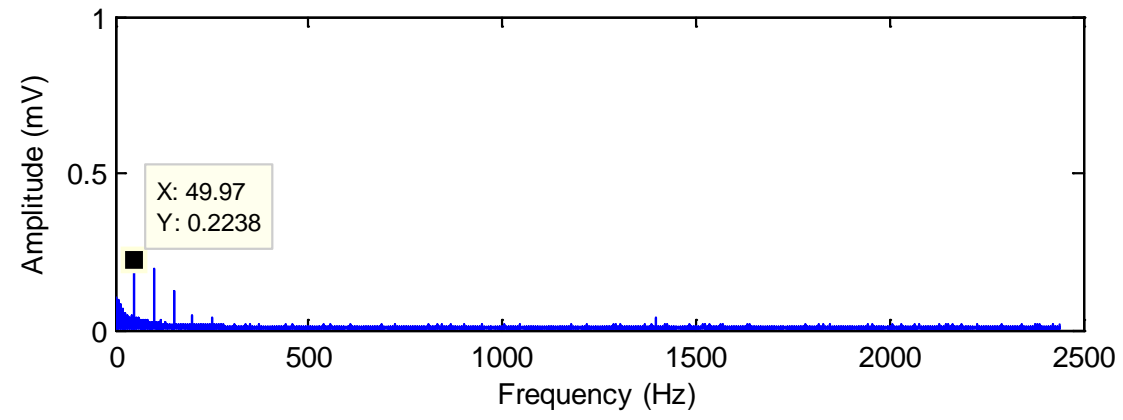

Fig. 6. Vibration data acquired on 24 February: (a) raw vibration data; (b) FFT.

According to the results, it can be concluded that the fault frequencies listed in Table 2 are difficult to identify using the FFT from the incipient defect (after dust insertion) to complete failure. One piece of information which can be identified from the raw vibration data is that the amplitude of the vibration signal increased gradually from February 24, May 3 to August 30 shown in Figs. 6(a) - 8(a). As FFT-based vibration is not appropriate, the feature extraction method was then considered. 
a

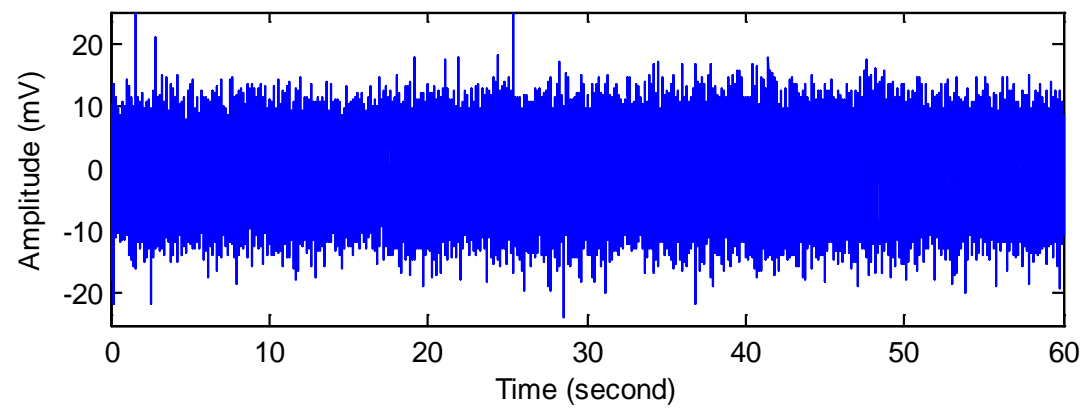

b

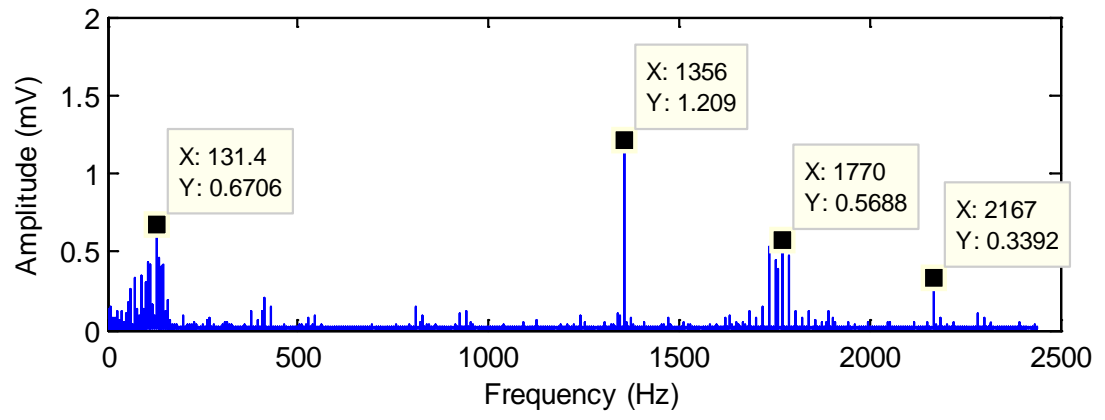

Fig. 7. Vibration data acquired on 3 May: (a) raw vibration data; (b) FFT.

a

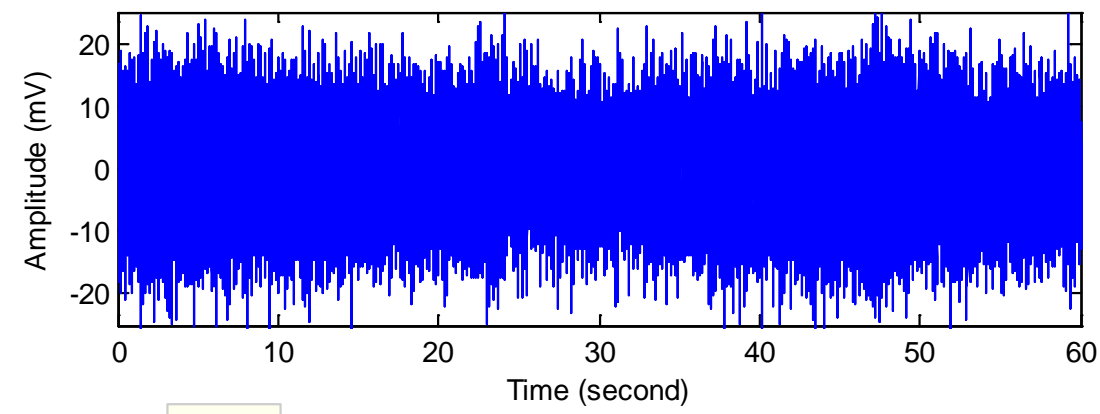

b

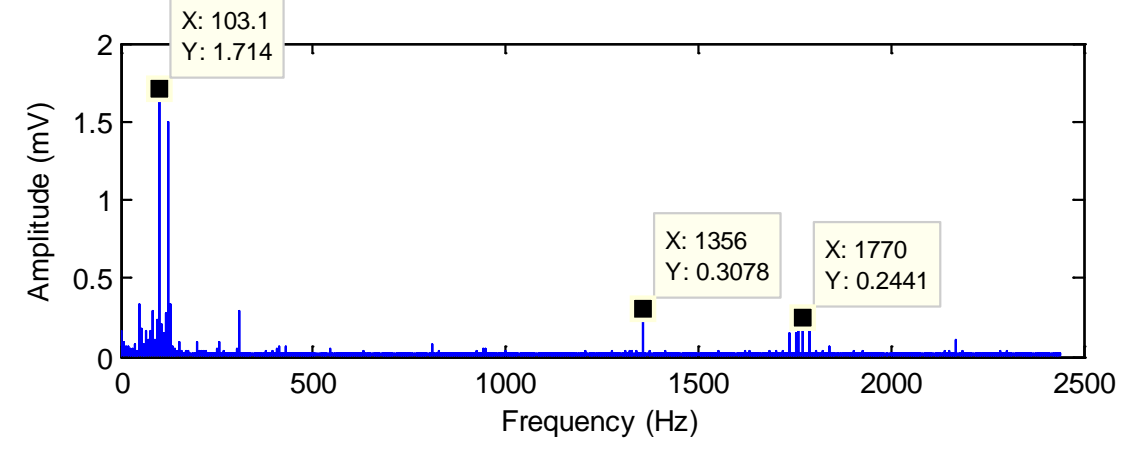

Fig. 8. Vibration data acquired on 30 August: (a) raw vibration data; (b) FFT.

\subsection{Time domain feature extraction}

As seen in the previous section, the vibration-based FFT is unable to provide the information related to the changes in the bearing condition, so the common time domain features such as mean, 
root mean square (RMS), shape factor, crest factor, skewness, kurtosis and entropy are extracted from the raw vibration signal of the slew bearing. These features have been applied for vibrationbased condition monitoring and have shown superior performance [17]. Recent study shows the application of the aforementioned features in low speed slew bearings [6]. According to the feature extraction result presented in [6], the changes in the condition of the slew bearing are not clearly visible in the mean, the RMS or the skewness feature. Only kurtosis can better show the changes in the bearing condition than those features. In this paper, the RMS, the skewness and the kurtosis are extracted from 10 seconds of vibration signal. The extracted features for 139 days of measurement are presented in Fig. 9. Due to the unsatisfactory result of the time domain features, the advanced signal processing method, empirical mode decomposition (EMD), is used.

a

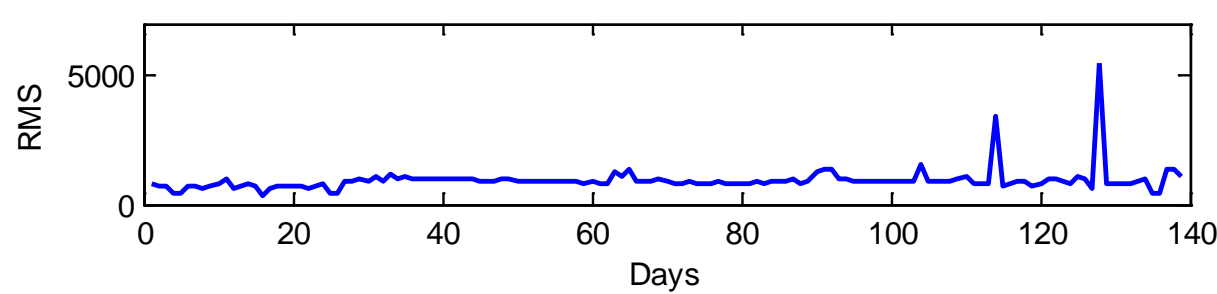

b

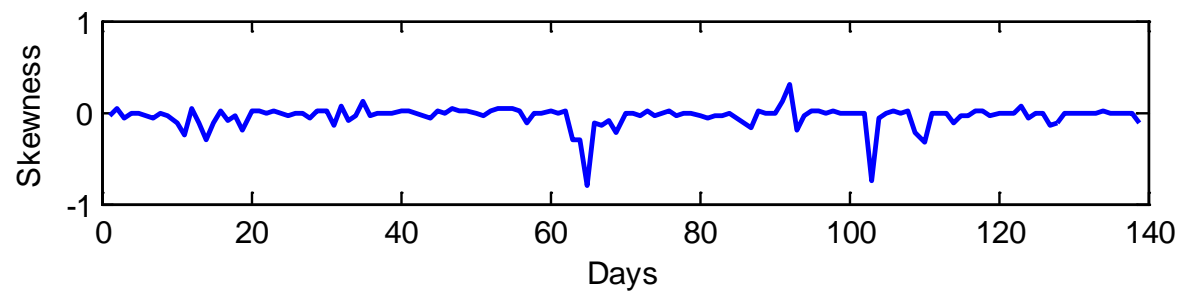

C

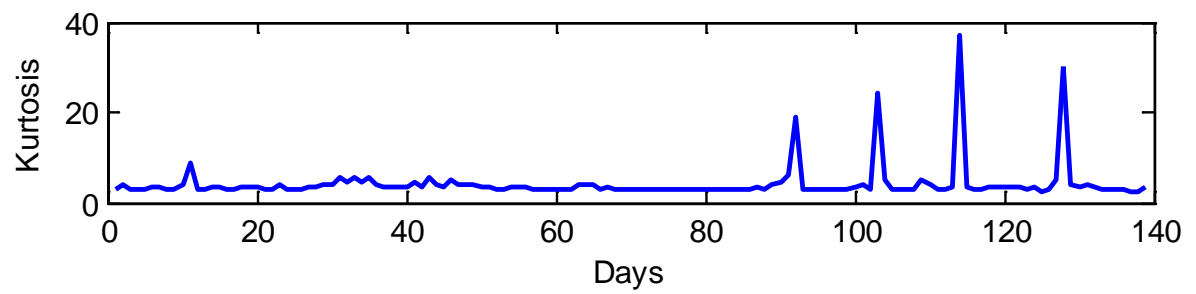

Fig. 9. Time domain features: (a) Root mean square (RMS); (b) skewness and (c) kurtosis.

\subsection{EMD-based feature extraction}

EMD [19] has been demonstrated to be useful in a wide variety of applications for extracting signals from data generated in noisy non-linear and non-stationary processes [20]. Recently, EMD has been applied for slew bearing data as presented in [21, 22]. In [21], the open source EMD MATLAB program developed by Rilling et al. [23] is utilized to decompose the slew bearing signal into several frequency components called intrinsic mode functions (IMFs). Since the low frequency components are important in the case of slew bearings, the results of IMF with low frequency 
content are then compared to the bearing fault frequencies presented in Table 2. Similar to the method presented in section 4.1, the three distinct data acquired on different days, namely February 24, May 3, and August 30, are used. According to the results, EMD was able to reveal bearing fault frequencies such as: a rolling element fault frequency of $0.43 \mathrm{~Hz}$ on May 3 and a bearing inner race fault of $1.37 \mathrm{~Hz}$ on August 30 [21]. In [22], the EMD is used as a comparison method for circular domain features. The selected EMD results of slew bearing data acquired on March 1, May 25 and August 30 are presented in Table 3. It is worth noting that the EMD result in this paper can also be found in [22]. It can also be seen that one identical fault frequency presented in Table 2 appeared in one of the IMF's results and is highlighted in yellow. Although the EMD is able to reveal the bearing fault frequency component and the result is better than the vibration-based FFT method, the result is not consistent with day-to-day measurement e.g. the frequency of $0.459 \mathrm{~Hz}$ which occurred on May 25 at IMF 12 did not appear again on August 30. Since the low frequency components are of the utmost importance in low speed slew bearings, the sum of these low IMF frequencies is used for feature extraction to identify whether or not the feature can show the bearing condition changes between February and August. Using EMD, the unwanted high frequency noise signals can also be removed and only the bearing signal with low frequencies is used for further feature extraction. We expected that the EMD-based feature extraction result would be better than the feature extraction of the raw vibration signal presented in section 4.2. The result of EMD-based feature extraction is presented in Fig. 10.

It can be seen from Fig. 10 that features extracted from the EMD has almost the identical pattern to the features extracted from the raw vibration data. In addition, the skewness of EMD is better that the skewness of the raw vibration signal. Despite the fact that the features presented in section 4.2 and 4.3 show some fluctuation in the last measurement period, approximately from July to August (from day 90 to 139), the trend is not increased. As a consequence, the progressive deterioration is difficult to identify based only on the features extracted from raw vibration data and the features extracted from EMD. 
Table 3. EMD results of three vibration data [22].

\begin{tabular}{lccc}
\hline \multirow{2}{*}{ IMF } & \multicolumn{3}{c}{ EMD results of three vibration data $(\mathrm{Hz})$} \\
\cline { 2 - 4 } & March 1 $^{\text {st }}$ & May 25 $^{\text {th }}$ & August 30 \\
\hline IMF 1 & 703.511 & 667.759 & 651.717 \\
IMF 2 & 688.399 & 550.533 & 679.863 \\
IMF 3 & 339.951 & 270.841 & 245.924 \\
IMF 4 & 135.495 & 132.749 & 121.320 \\
IMF 5 & 84.989 & 68.341 & 63.555 \\
IMF 6 & 41.812 & 34.238 & 28.892 \\
IMF 7 & 20.336 & 17.494 & 13.682 \\
IMF 8 & 8.183 & 8.620 & 6.465 \\
IMF 9 & 3.973 & 4.270 & 3.054 \\
IMF 10 & 2.058 & 28.112 & 1.374 \\
IMF 11 & 1.142 & 1.035 & 0.682 \\
IMF 12 & 0.544 & 0.459 & 0.332 \\
IMF 13 & 0.284 & 0.295 & 0.099 \\
IMF 14 & 0.100 & 0.099 & - \\
IMF 15 & - & 0.096 & - \\
\hline
\end{tabular}

a

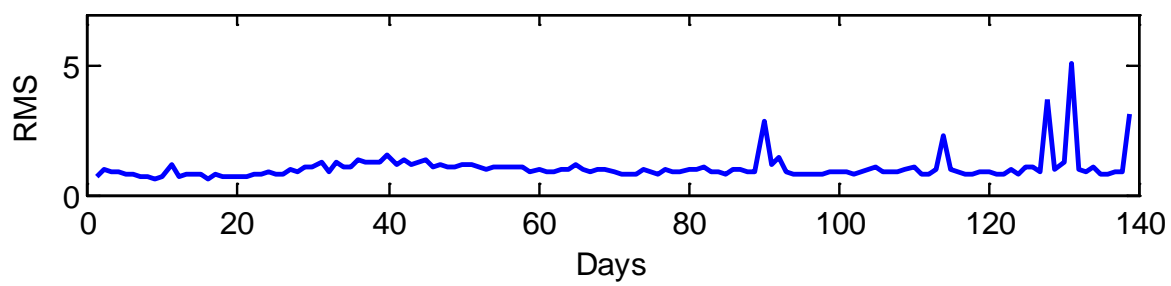

b

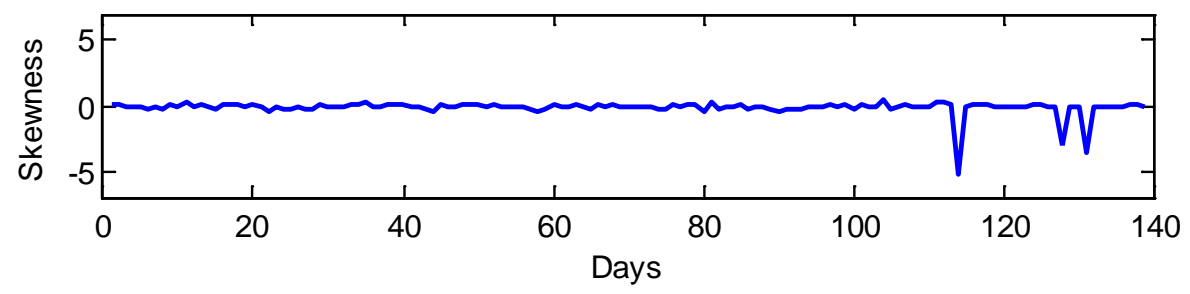

c

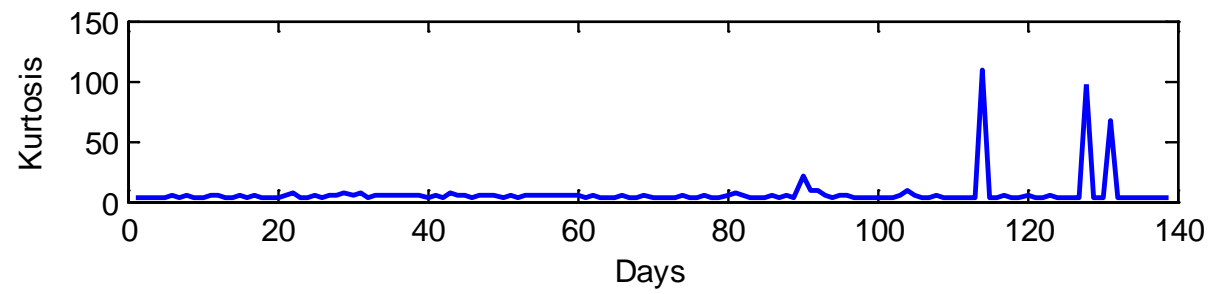

Fig. 10. Features extracted from EMD: (a) RMS; (b) skewness and (c) kurtosis. 


\subsection{LLE feature}

\section{LLE algorithm for one second vibration data}

The objective of using the LLE algorithm in this paper is to identify the onset of the deterioration of the bearing. As the slew bearing rotates at very low rotational speed $(\approx 1 \mathrm{rpm})$, the bearing signal generated from the contact between the rolling element and the defective point is very weak and buried in unwanted noise, thus the onset of the deterioration in the condition of the bearing and the progression of the deterioration cannot be clearly identified as shown in the extracted features of the raw vibration data presented in section 5.2 and the extracted features from EMD as presented in section 5.3.

The total data length for laboratory slew bearing data is 60 seconds multiplied by the 4880 samples produced 292800 samples acquired at the same time each day. In the first application of the LLE algorithm for laboratory slew bearing data, the 30 second data containing 146400 samples was processed by the LLE algorithm. To identify non-linear characteristic of the vibration data, every one second containing 4880 samples $(N=4880)$ was inputted into the LLE algorithm. The illustration of the LLE algorithm applied at one second intervals is presented in Fig. 11. The result is the LLE feature denotes by $\lambda_{1}$. As can be seen in the table in Fig. 11, the result of the LLE feature $\left(\lambda_{1}\right)$ can be positive or negative depending on the input data at a certain time (second). The detail $\lambda_{1}$ result for each second during the 30 second vibration signal is depicted in Table 4. It can be seen from Fig. 11 and Table 4 that during 30 second' measurement in one day, the majority of the LLE results are negative $\lambda_{1}\left(-\lambda_{1}\right)$ and only a few of them are positive $\lambda_{1}\left(+\lambda_{1}\right)$. The negative or positive $\lambda_{1}$ is the computational result of Eq. (13), where, negative $\lambda_{1}$ indicates the newly measured distance $\mathbf{d}_{\text {new }}(i)$ is unchanged or fixed. The physical interpretation of negative $\lambda_{1}$ is given at the $9^{\text {th }}$ second of the vibration data on April 26, as shown in Fig. 11(a). If, instead, the newly measured distance $\mathbf{d}_{\text {new }}(i)$ increases exponentially, the value of $\lambda_{1}$ is positive. The physical interpretation of positive $\lambda_{1}$ is given at the $16^{\text {th }}$ second, as shown in Fig. 11(b). In addition, the negative $\lambda_{1}$ means the processed vibration data at one particular second is in a stable or normal category, while positive $\lambda_{1}$ indicates the vibration data is non-linear. As time progresses and the condition of the slew bearing deteriorates or an unsustainable fault develops, it is expected that the count of positive $\lambda_{1}$ will increase. This is shown by the plotted count of positive $\lambda_{1}$ against 139 days' measurement as shown in Fig. 12. It can be seen that in the last measurement period (i.e. August 2007), the count of positive $\lambda_{1}$ increases significantly. This indicates the vibration data on August 2007 is more non-linear than in the previous months. 
The LLE parameters to obtain the LLE features $\left(\lambda_{1}\right)$, as shown in Fig. 11, are determined as follows: It has been mentioned in section 2 that $J$ is equal to 48. This value is determined based upon the dominant frequency of the vibration signal and the number of samples $N$. From Fig. 8(b), it can be seen that the dominant frequency of the vibration signal is $103.1 \mathrm{~Hz} . J$ is calculated as follows: $(1 / 103.1 \mathrm{~Hz})$ multiplied by 4880 samples $=47.33$ (rounding it up to the nearest integer, thus $J=48$ ). For selection of the $\mu$, it is recommended to remove the first sinusoidal cycle of $\mathbf{d}_{0}(i)$ which is the nearest neighbor of zero value at particular $i$, as shown, for example, in Fig. 2 (at $i=1$ ). This is because the first sinusoidal cycle that is close to zero value at particular $i$ typically demonstrates some transient behavior. Thus, $\mu$ is selected as 50 . In addition, to have faster computation time, $m$ is determined empirically to get $M$ as low as possible (note that it is possible to get a negative $M$ value if the combination of the value $m$ and $J$ are inappropriate). If $J=48$, the optimal $m=100$, using the equation $M=N-(m-1) J, M=128$. If $J=48$ and $m=110, M$ will be negative $(M=-352)$. On the other hand, if $J$ is still 48 and $m$ is decreased ( $m=90$ ), the value of $M$ is increased to about 608 (if $M$ is higher, the computational time will be slower).

Table 4. The LLE result $\left(\lambda_{1}\right)$ at one second intervals during the 30 second vibration signal (data April 26).

\begin{tabular}{cccc}
\hline Time (sec) & LLE result $\left(\lambda_{1}\right)$ & Time $(\mathrm{sec})$ & LLE result $\left(\lambda_{1}\right)$ \\
\hline 1 & -7.3658 & 16 & 12.9481 \\
2 & -61.2420 & 17 & -24.1665 \\
3 & -20.6105 & 18 & -33.0387 \\
4 & -62.2330 & 19 & -37.3419 \\
5 & -75.1535 & 20 & -53.4996 \\
6 & 11.2430 & 21 & -9.4541 \\
7 & -52.0686 & 22 & -63.5334 \\
8 & -49.3602 & 23 & -10.2493 \\
9 & -10.1467 & 24 & -65.9973 \\
10 & -60.8948 & 25 & -24.5865 \\
11 & -2.9959 & 26 & -36.1825 \\
12 & -74.9254 & 27 & -7.0326 \\
13 & -52.4728 & 28 & -50.9032 \\
14 & -49.9062 & 29 & -6.5783 \\
15 & -101.4999 & 30 & -21.7180 \\
\hline
\end{tabular}



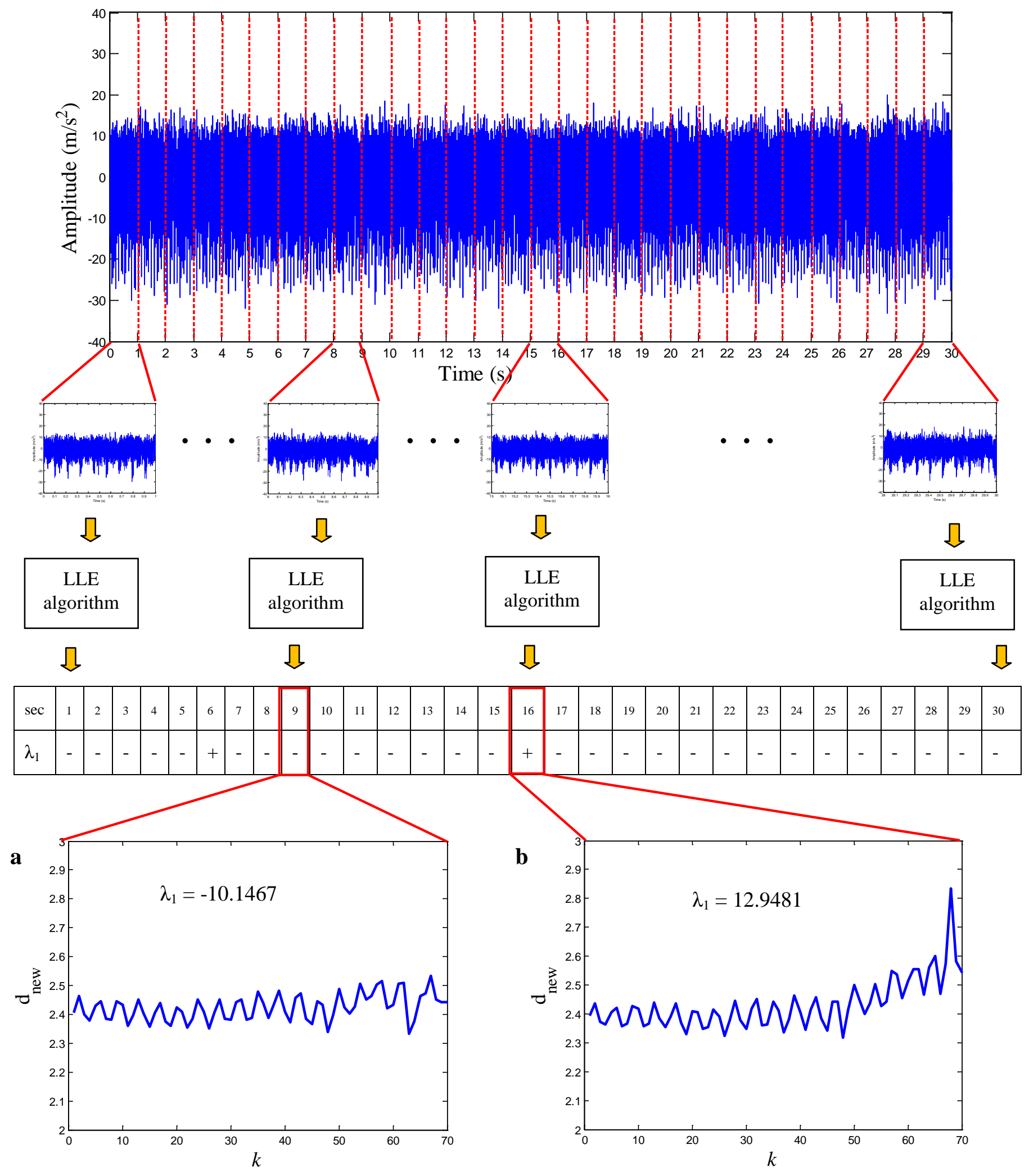

Fig. 11. One day LLE result (data April 26): (a) at the $9^{\text {th }}$ second (stable condition, negative $\lambda_{1}$ ); (b) at the $16^{\text {th }}$ second (non-linear condition, positive $\lambda_{1}$ ). 


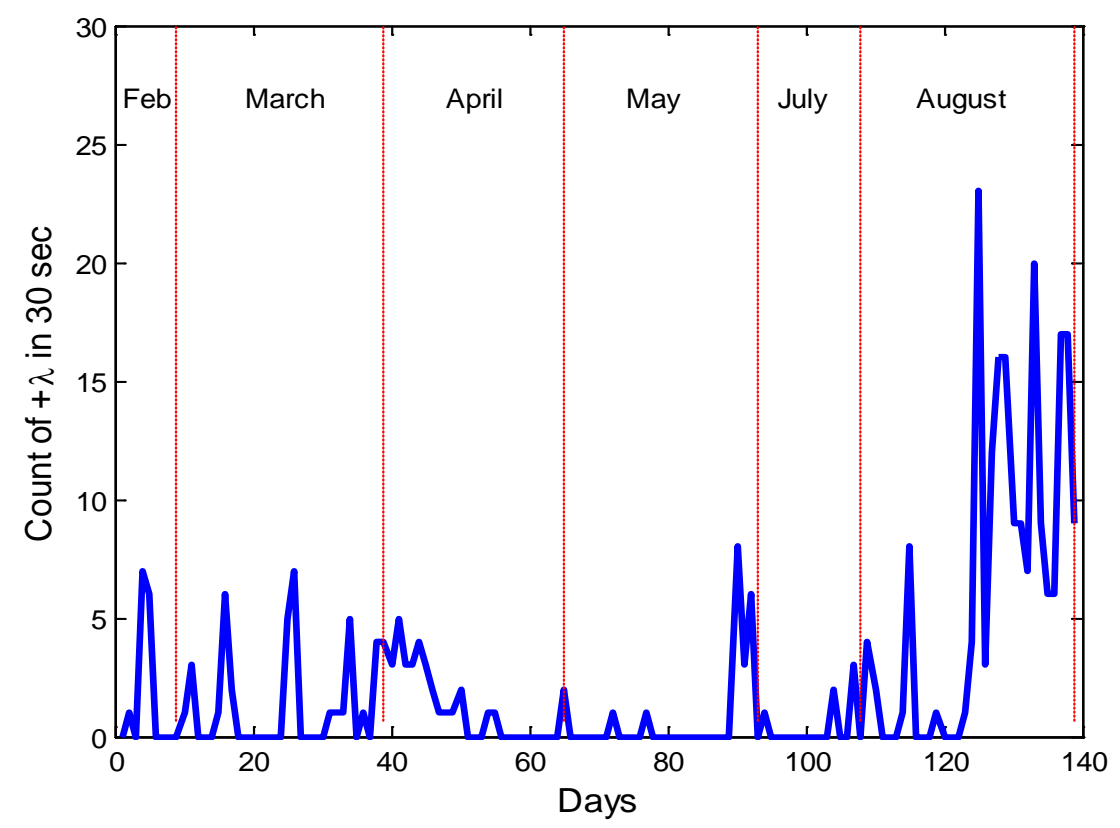

Fig. 12. Number of positive $\lambda_{1}$ over the 139 measurement days from February to August 2007.

\section{LLE algorithm for ten second vibration data}

One practical challenge in monitoring low speed slew bearings is the large amount of data acquired over a long duration (e.g. 30 to 60 seconds). An efficient method that can be applied for only short duration vibration data is, therefore, necessary. The method must also be able to provide rapid condition monitoring information for maintenance engineers to make the computational time faster. Instead of using the 30 second or 60 second vibration data, 10 second vibration data is used. The LLE algorithm is applied to every one second, as shown previously, but in this case, the duration of the vibration data used in only 10 seconds. The maximum $\left(\lambda_{1}\right)$ is collected from 10 results for each day. To explain the advantage of the proposed method, the LLE feature (largest $\lambda_{1}$ ) extracted from the 10 second vibration data each day during 139 measurement days is plotted in the same figure with other comparable methods: kurtosis feature extracted from raw vibration data and kurtosis feature extracted from EMD, as shown in Fig. 13(a). The LLE feature is normalized to the minimum and maximum values of the kurtosis feature extracted from raw vibration data before it is plotted in the same figure. Although the three comparable kurtosis features presented in Fig. 13(a) show fluctuation in the last measurement days which indicates changes in the condition of the bearing, the LLE has an additional benefit. It can be seen that the LLE feature also increases exponentially over the last measurement days (approximately from day 110 to day 139) indicating the increased deterioration. Such an exponential trend cannot be extracted using time domain 
features extracted from raw vibration signals and features extracted from EMD. It should be noted that the increased progression deterioration is a warning of complete bearing failure. The LLE feature is supported by the inspection of the slew bearing damage after day 139 as shown in Fig. 14. Some defective regions in the roller element and the outer race can be clearly seen in Fig. 14.

a

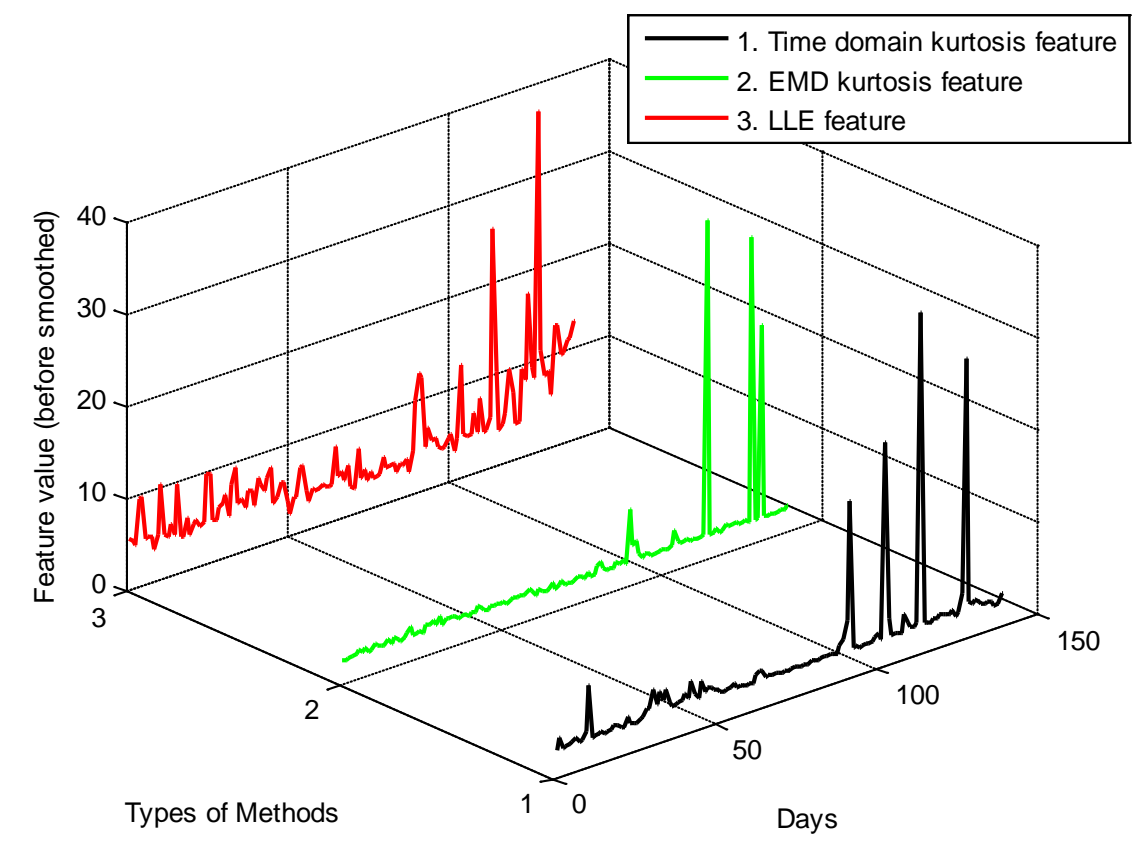

b

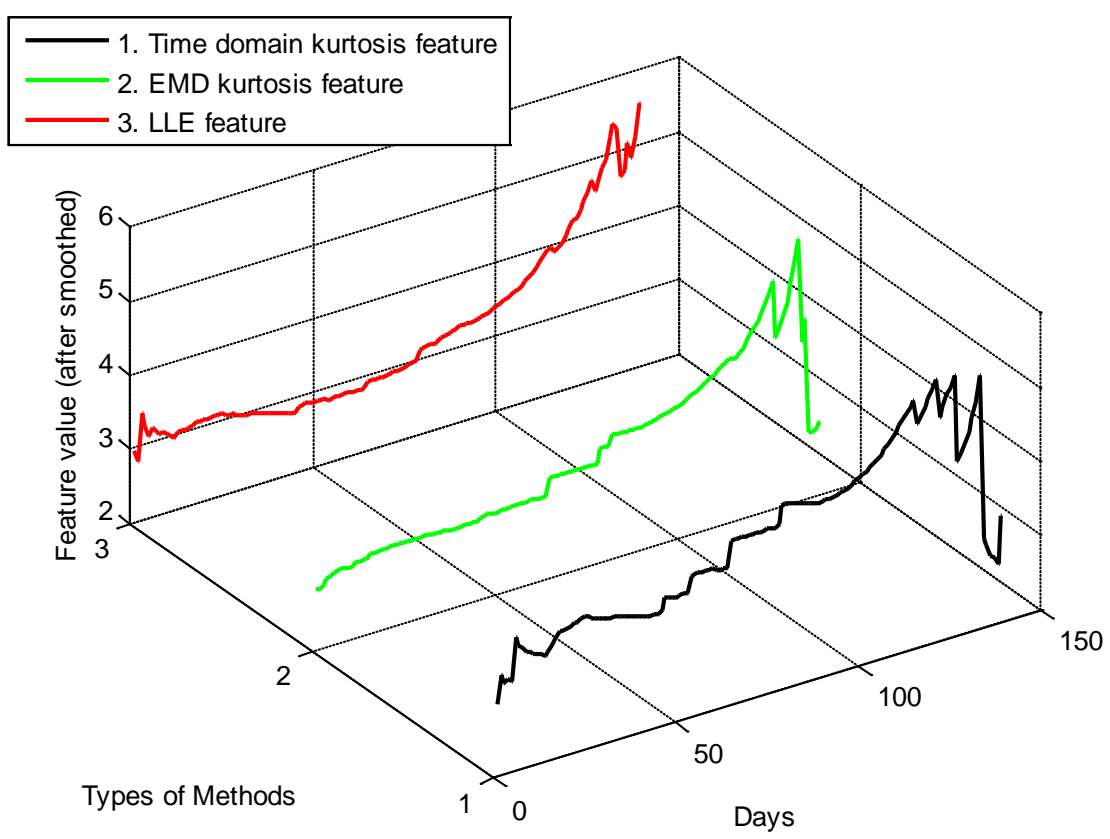

Fig. 13. Comparison condition monitoring performance between the LLE feature and other features: time domain features of raw vibration data and features of EMD: (a) features before smoothing; (b) features after smoothing of 100 samples. 
To present clearly the progression of the deterioration, a smoothing technique is used. The standard smoothing technique of 100 samples is employed. Using a moving window, an average of 100 samples is calculated. The moving window progresses from 1, 2, ., 139. It can be seen from Fig. 13(b) that the LLE feature after smoothing is increased exponentially. In contrast, the kurtosis of the raw vibration signal and the kurtosis of EMD did not increase exponentially and suddenly dropped over the last few days.
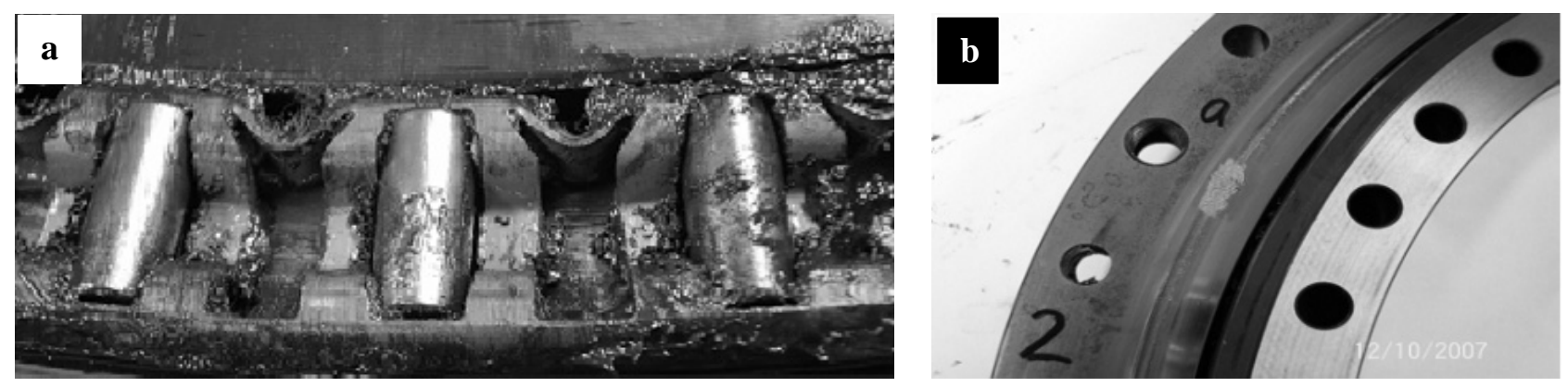

Fig. 14. Slew bearing damage: (a) a view of damaged rollers in the axial row; (b) outer raceway damage.

\section{Results and discussion of coal bridge reclaimer data}

\subsection{Vibration-based FFT}

Similar to vibration based monitoring discussed in the laboratory slew bearing test rig section, the FFT is calculated initially from all 45 raw vibration data sets acquired from a slew bearing installed in a coal bridge reclaimer used to support the production of a local steel mill. The 45 raw vibration data sets were collected once a month from May 2003 to November 2006. The detail of 45 measurement dates of vibration data sets are presented in Table 5. The example of FFT results calculated from three different measurement dates are shown in Fig. 15. The three different measurement dates for FFT are 28 July 2003, 16 September 2004 and 14 September 2005. It can be seen from Fig. 15 that there are at least 5 dominant frequencies appearing: $16.7 \mathrm{~Hz}, 23.44 \mathrm{~Hz}, 33.34$ $\mathrm{Hz}, 50.04 \mathrm{~Hz}$ and $66.75 \mathrm{~Hz}$. Those dominant frequencies are above the fault frequencies shown in Table 6. Although the exact age of the bearing was not provided, the vibration data collected on 1 May 2003 (the first measurement) indicated that it was still in a normal condition. The coal bridge reclaimer was located in the open air and subjected to the elements, including all the various forms of dust particles that are part of a steelmaking complex. Because the vibration data were acquired in a dusty environment, it was supposed that the bearing was in fault mode (even if at a low level of fault) after having run continuously for more than two years. Thus, the FFT of the vibration data on 14 September 2005, as shown in Fig. 15(c), should have the dominant frequencies which match 
bearing fault frequencies. However, it was difficult to detect the fault frequencies of slew bearing using FFT as shown in Fig. 15. In addition, it was found that none of the dominant frequencies of FFT shown in Fig. 15 matched with the fault frequencies depicted in Table 6. This indicates that the level of fault is very low and masked by the background noise. Further, feature extraction methods based on time domain features and the LLE feature are applied to the similar raw vibration data.

Table 5. Detailed measurement dates of coal bridge reclaimer data

\begin{tabular}{cccccc}
\hline $\begin{array}{c}\text { Measurement } \\
\text { day }\end{array}$ & Date & $\begin{array}{c}\text { Measurement } \\
\text { day }\end{array}$ & Date & $\begin{array}{c}\text { Measurement } \\
\text { day }\end{array}$ & Date \\
\hline 1 & 1 May 2003 & 16 & 29 Jun 2004 & 31 & 12 Aug 2005 \\
2 & 28 May 2003 & 17 & 22 Jul 2004 & 32 & 14 Sep 2005 \\
3 & 24 Jun 2003 & 18 & 19 Aug 2004 & 33 & 13 Oct 2005 \\
4 & 28 Jul 2003 & 19 & 16 Sep 2004 & 34 & 28 Nov 2005 \\
5 & 19 Aug 2003 & 20 & 14 Oct 2004 & 35 & 22 Dec 2005 \\
6 & 18 Sep 2003 & 21 & 29 Oct 2004 & 36 & 27 Jan 2006 \\
7 & 16 Oct 2003 & 22 & 18 Nov 2004 & 37 & 28 Feb 2006 \\
8 & 13 Nov 2003 & 23 & 16 Dec 2004 & 38 & 30 Mar 2006 \\
9 & 12 Dec 2003 & 24 & 13 Jan 2005 & 39 & 27 Apr 2006 \\
10 & 8 Jan 2004 & 25 & 8 Feb 2005 & 40 & 25 May 2006 \\
11 & 5 Feb 2004 & 26 & 3 Mar 2005 & 41 & 30 Jun 2006 \\
12 & 16 Mar 2004 & 27 & 7 Apr 2005 & 42 & 28 Jul 2006 \\
13 & 1 Apr 2004 & 28 & 12 May 2005 & 43 & 7 Sep 2006 \\
14 & 5 May 2004 & 29 & 2 Jun 2005 & 44 & 31 Oct 2006 \\
15 & 7 Jun 2004 & 30 & 7 Jul 2005 & 45 & 30 Nov 2006 \\
\hline
\end{tabular}

Table 6. Fault frequencies of slew bearing coal bridge reclaimer run at $4.5 \mathrm{rpm}$ [calculation is given in Appendix A]

\begin{tabular}{lcc}
\hline \multirow{2}{*}{ Defect mode } & \multicolumn{2}{c}{ Fault frequencies $(\mathrm{Hz})$} \\
\cline { 2 - 3 } & Axial & Radial \\
\hline Outer ring (BPFI) & 13.41 & 11.38 \\
Inner ring (BPFO) & 13.58 & 11.56 \\
Rolling element (BSF) & 5.65 & 4.87 \\
\hline
\end{tabular}



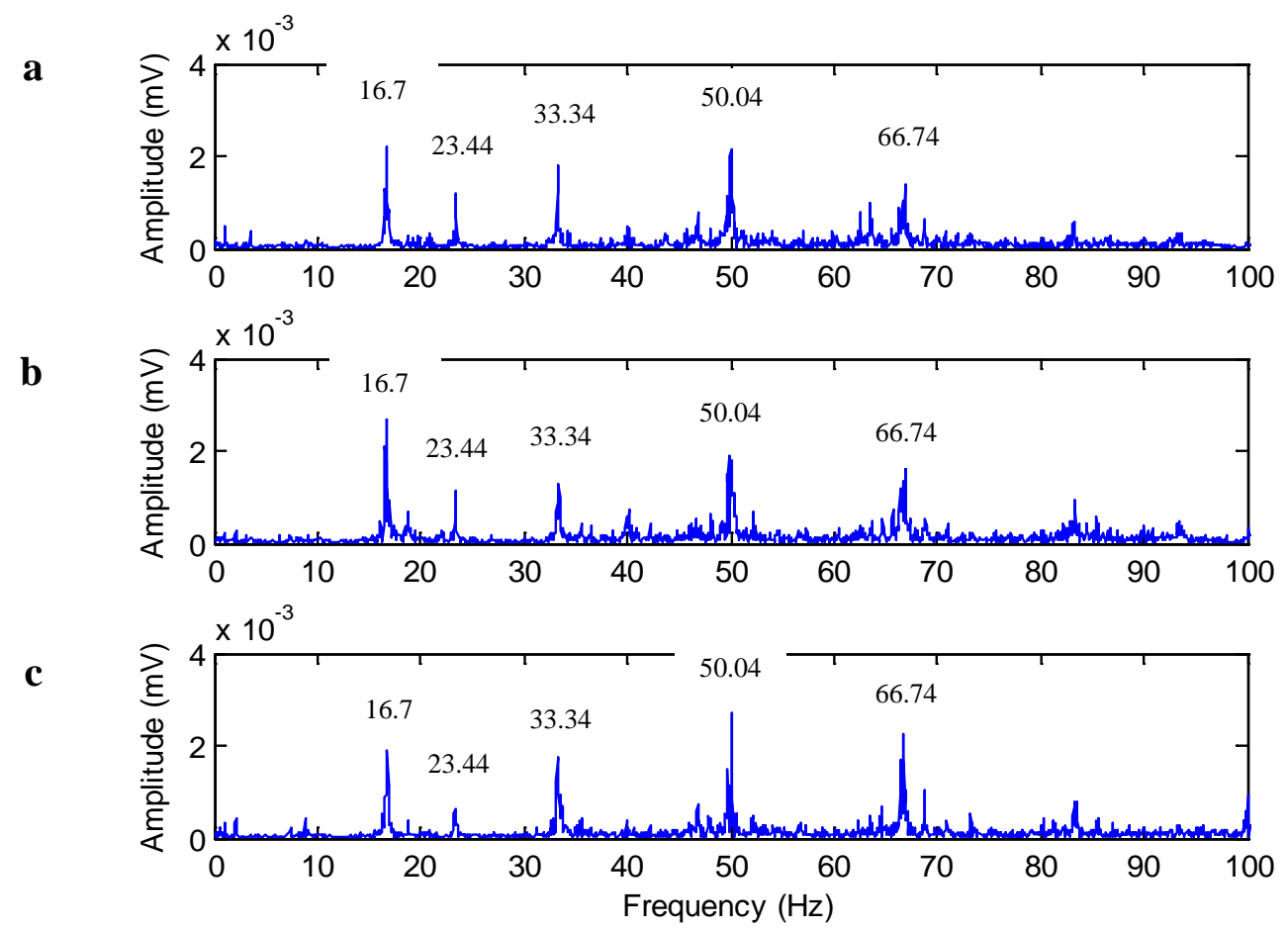

Fig. 15. FFT of coal bridgre reclaimer: (a) data 28 July 2003; (b) data 16 September 2004 and (c) data 14 September 2005.

\subsection{Feature extraction (time domain and LLE feature)}

The RMS, skewness and kurtosis features extracted from 45 vibration data sets of the slew bearing coal bridge reclaimer are presented in Fig. 16. Because the slew bearing is located in an open environment, the degradation of the bearing is expected to increase during the approximately 3.5 year measurement from May 2003 to November 2006. However, the RMS, skewness and kurtosis features did show such a condition. It can be seen from Figs. 16(a) to (c) that the RMS, skewness and kurtosis features did not show the progressive deterioration over 3.5 years. The only information that can be extracted is the two high peaks appear in the skewness and the kurtosis feature. The LLE algorithm was then applied to the similar data.

In contrast to the result of the time domain features, the LLE feature $\left(\lambda_{1}\right)$ presented in Fig. 16(d) shows the significant increase on the last measurement dates, especially in measurement 44 and measurement 45. It also clearly seen that the two high peaks which are identified on the skewness and the kurtosis feature were also appear in the LLE feature. The first peak indicates the onset of deteriorating bearing condition and the second peak shows the deterioration of the bearing will increase in the future. Additional information can also be found from the LLE feature that the 
second peak is greater that the first peak. This indicates that LLE feature can extract more information regarding to the development of bearing deterioration. In contrast, the second high peak of the time domain kurtosis is remaining in the same level with the first high peak which is difficult to evaluate the bearing deterioration. Unfortunately, the measurement had to stop at measurement 45, which is 30 November 2006, due to the commencement of preparation for the acquisition of vibration data from the laboratory slew bearing test rig. The LLE feature of the bridge reclaimer data shows that the actual vibration slew bearing data is more non-linear than the laboratory slew bearing vibration data. It is proved by the fact that the number of positive $\lambda_{1}$ is greater than the number of negative $\lambda_{1}$. In the beginning, there is negative $\lambda_{1}$, but as time progresses, the $\lambda_{1}$ will swift become positive. As wear developed, the value of the positive $\lambda_{1}$ will increase correspondently.

The LLE parameters to obtain the LLE features $\left(\lambda_{1}\right)$ as shown in Fig. 16(d) and Table 7 are determined as follows: based on the known sampling rate of 240 samples per second acquired for 17.067 seconds, 4096 samples were produced. The first step for the LLE algorithm is to determine the variables $J$ and $m$. Once $J, m$ and $N$ are known, $M$ can be calculated. As shown in section 5.4, $J$ can be calculated based upon the first dominant frequency of the vibration signal being analyzed. As shown in Fig. 15, the first dominant frequency is $16.7 \mathrm{~Hz}$. Thus $J$ is calculated in the following way: (1/16.7 Hz) multiplied by $4096 \mathrm{~Hz}=14.37$. The numerical computation required the nonnegative integer of $J$ and also required that $J$ should be the element of factorial 4096. Thus we round up 14.37 to greater integer number 16 . The integer 16 is selected because it an element of factorial 4096. The reason why it needs to be an element of factorial 4096 is because of the phase space requirement. If $J$ is an element of factorial 4096, embedding dimension $m$ will be easy to calculate. The next variable which needed to be determined once $J$ is known is $m$. As shown in section 5.4, $m$ is selected to get the $M$ as low as possible in order to speed up the computation time. The computation time of the feature extraction method is necessary in practice to provide a rapid condition monitoring result. Thus $m$ in this paper for the coal bridge reclaimer data is 248 . The variables $\mu$ and $k$ are selected as 30 and 40 , respectively. 
a

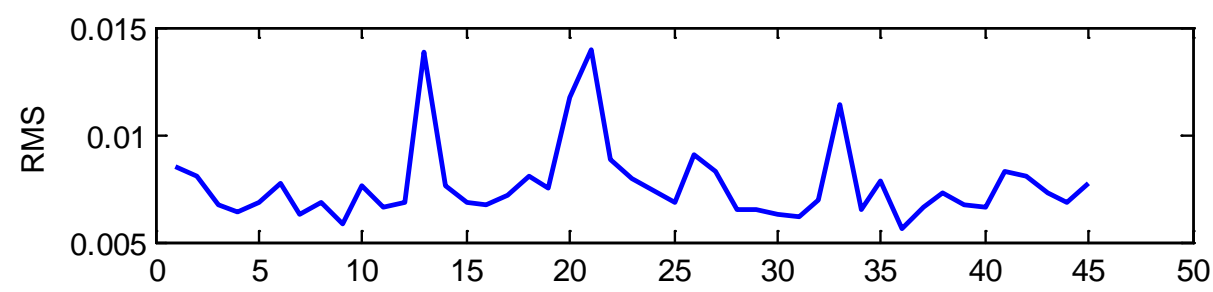

b

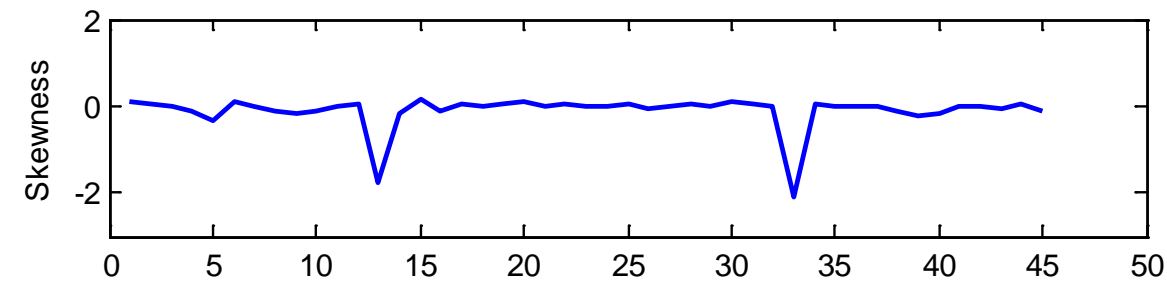

c

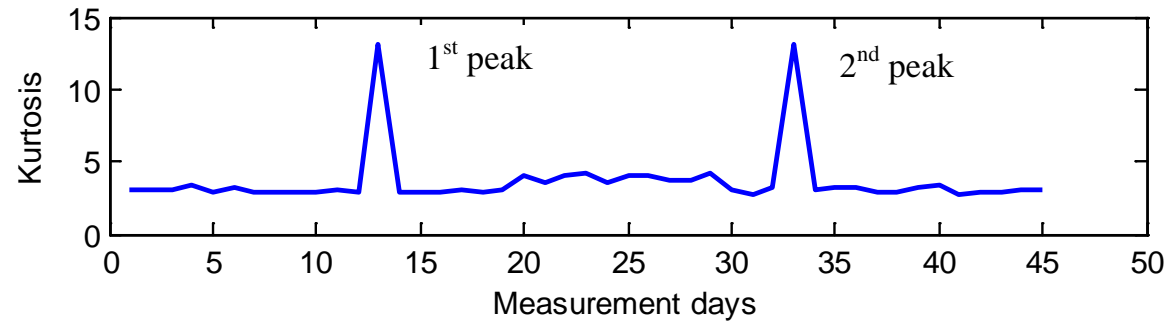

d

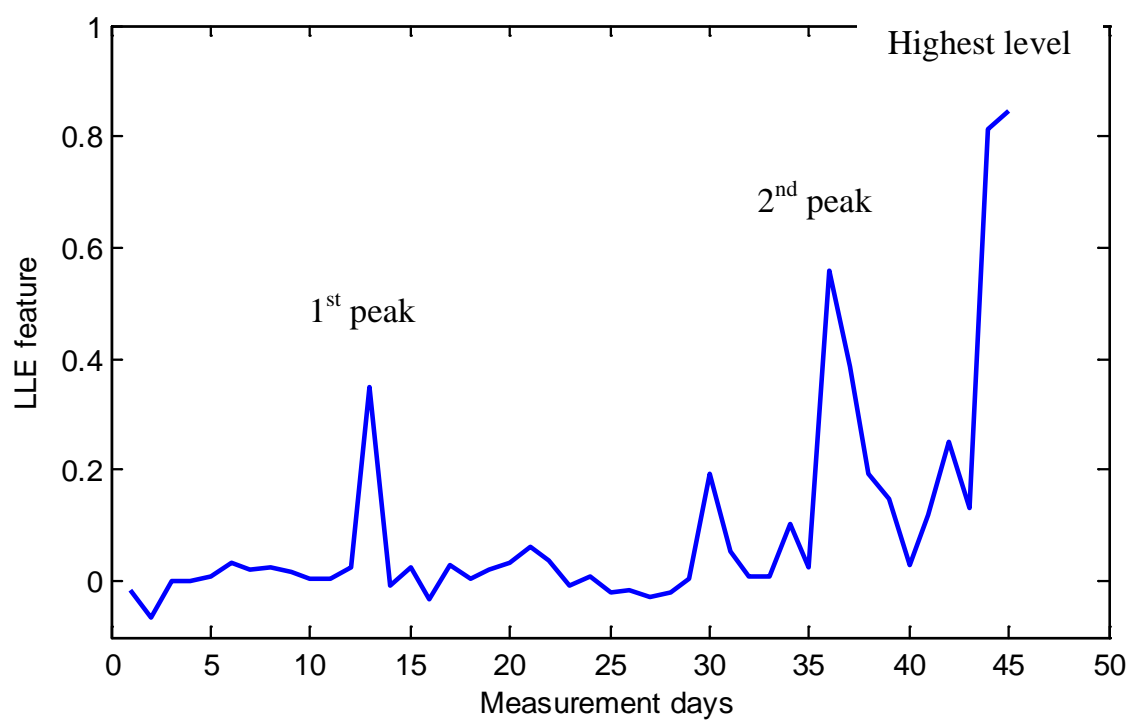

Fig. 16. Comparison between time domain features and LLE feature extracted from the coal bridge reclaimer data: (a) RMS feature; (b) skewness feature; (c) kurtosis feature and (d) LLE feature. The measurement in the $x$-axis is related to Table 5 . 
Table 7. Detailed of LLE result $\left(\lambda_{1}\right)$ related to the measurement dates

\begin{tabular}{|c|c|c|c|c|c|c|c|}
\hline \multicolumn{2}{|c|}{$\begin{array}{c}\text { Measurement } \\
\text { day }\end{array}$} & $\lambda_{1}$ & \multirow{2}{*}{$\begin{array}{c}\text { Measurement } \\
\text { day } \\
16\end{array}$} & \multirow{2}{*}{$\begin{array}{c}\lambda_{1} \\
-0.031\end{array}$} & \multirow{2}{*}{$\begin{array}{c}\begin{array}{c}\text { Measurement } \\
\text { day }\end{array} \\
31\end{array}$} & \multirow{2}{*}{$\begin{array}{c}\lambda_{1} \\
0.05518\end{array}$} & \\
\hline & 1 & -0.01667 & & & & & \\
\hline & 2 & -0.06506 & 17 & 0.0296 & 32 & 0.01014 & \\
\hline & 3 & 0.0003934 & 18 & 0.002878 & 33 & 0.007898 & \\
\hline & 4 & -0.0008573 & 19 & 0.02163 & 34 & 0.1046 & $2^{\text {nd }}$ peak \\
\hline & 5 & 0.0106 & 20 & 0.03241 & 35 & 0.02563 & \\
\hline & 6 & 0.03377 & 21 & 0.06081 & 36 & 0.557 & \\
\hline & 7 & 0.01959 & 22 & 0.03731 & 37 & 0.3844 & \\
\hline & 8 & 0.02359 & 23 & -0.007276 & 38 & 0.1947 & \\
\hline & 9 & 0.01857 & 24 & 0.007093 & 39 & 0.1491 & \\
\hline & 10 & 0.005054 & 25 & -0.01873 & 40 & 0.0309 & \\
\hline & 11 & 0.004379 & 26 & -0.01511 & 41 & 0.1214 & 2 highest \\
\hline & 12 & 0.0271 & 27 & -0.02856 & 42 & 0.2491 & levels \\
\hline & 13 & 0.3485 & 28 & -0.02158 & 43 & 0.1337 & \\
\hline & 14 & -0.008846 & 29 & 0.004501 & 44 & 0.8137 & \\
\hline & 15 & 0.02722 & 30 & 0.1925 & 45 & 0.8475 & \\
\hline
\end{tabular}

\section{Evaluation criteria in tracking progressive slew bearing failure}

In this study, four evaluation criteria for tracking the progressive failure of low speed slew bearings were proposed. The criteria are:

1. Count of high peaks

2. High peak difference

3. Time of first peak detection

4. Interval between peaks

In order to substantiate the benefits of the LLE method, the four criteria above were applied to the time domain kurtosis feature, the EMD kurtosis feature and the LLE feature for laboratory slew bearing data and were also applied to the kurtosis and the LLE feature of coal bridge reclaimer data. The result is presented in Tables 8 and 9. The definition of and the formula for each evaluation criterion are described in Appendix B. In the case of laboratory slew bearing data, it can be seen that the overall evaluation score of the LLE feature was higher than the time domain kurtosis feature and the EMD feature. A negative of the $E_{2}$ criterion of EMD kurtosis feature is due to the anterior peaks are lower than the detection of the first peak. In the same way, the overall evaluation score of the LLE feature was greater than the kurtosis feature extracted from original vibration data for the case of coal bridge reclaimer data. 
Table 8. Evaluation comparison result for laboratory slew bearing data (Appendix B)

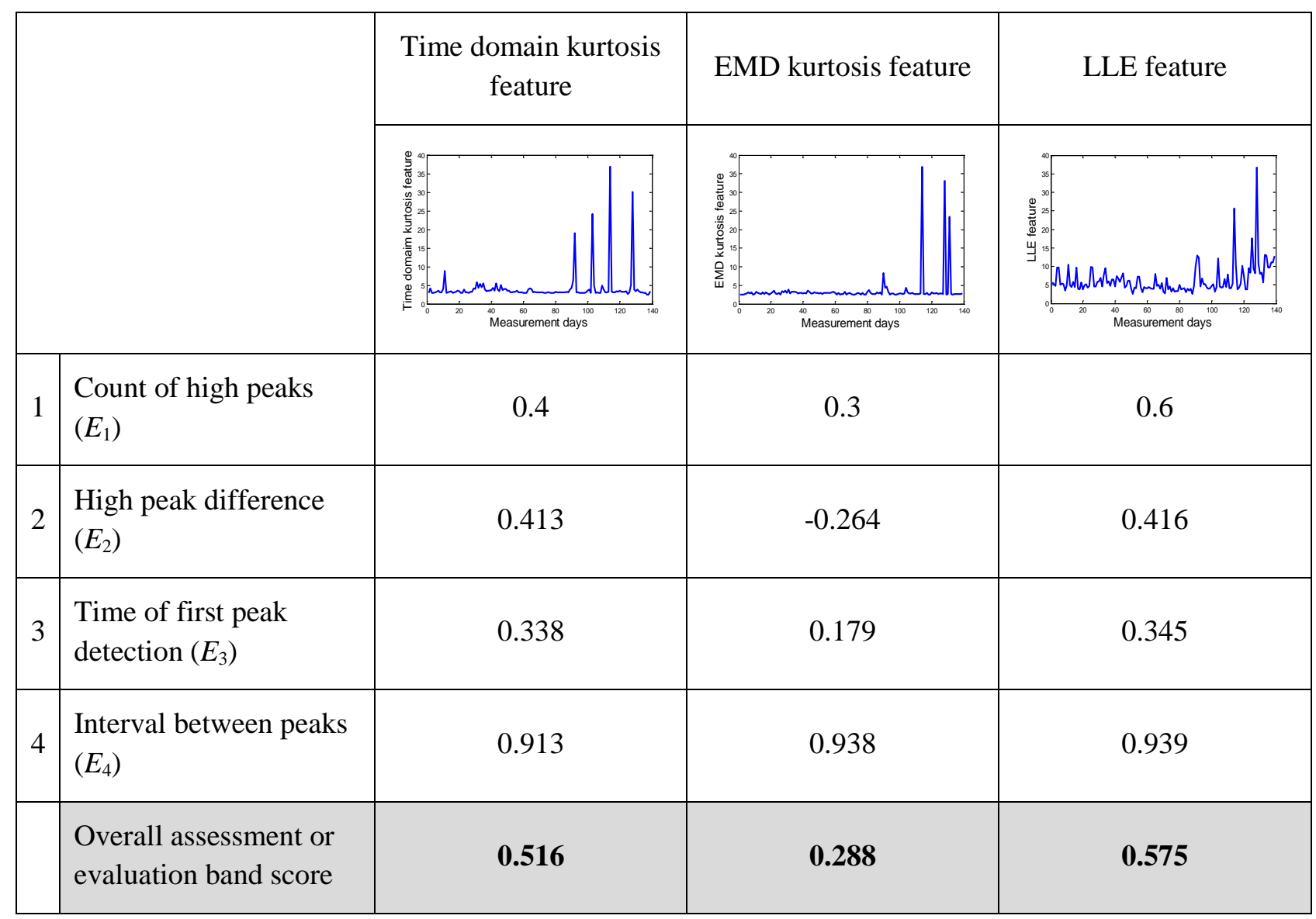

Table 9. Evaluation comparison result for coal bridge reclaimer data (Appendix C)

\begin{tabular}{|l|l|c|c|}
\hline \multicolumn{2}{|c|}{} & $\begin{array}{c}\text { Time domain kurtosis } \\
\text { feature }\end{array}$ & LLE feature \\
\cline { 3 - 4 } & & 0.2 & 0.5 \\
\hline 1 & Count of high peaks $\left(E_{1}\right)$ & 0.007 & 0.311 \\
\hline 2 & High peak difference $\left(E_{2}\right)$ & 0.711 & 0.711 \\
\hline 3 & Time of first peak detection $\left(E_{3}\right)$ & 0.555 & 0.822 \\
\hline 4 & Interval between peaks $\left(E_{4}\right)$ & $\mathbf{0 . 3 6 8}$ & $\mathbf{0 . 5 8 6}$ \\
\hline & Overall assessment or evaluation band score & \\
\hline
\end{tabular}




\section{Conclusion}

One of the phase space dissimilarity techniques called the largest Lyapunov exponent (LLE) algorithm has been employed as a feature extraction method for large low speed slew bearing of lab test condition monitoring and in a coal bridge reclaimer used in a local steel mill industry. According to the result, this method can be used as an alternative method to deal with low energy non-linear vibration bearing signals in cases where the existing methods which have been applied for rolling element bearing condition monitoring such as vibration-based FFT, time domain feature extraction (e.g. RMS, skewness, kurtosis, etc.), and advance fault diagnosis methods, e.g. empirical mode decomposition (EMD) are not suitable. The step-by-step computational algorithm has been presented in detail. It must be noted, that the most important LLE parameter is reconstruction delay $J$. This parameter must be calculated before the other parameters $m$ and $M$. This paper has also proposed a simple and effective technique to select parameter $J$. Four evaluation criteria has also been proposed and described to substantiate the benefits of LLE method.

\section{Acknowledgement}

The first author thanks the University of Wollongong for the financial support through University Postgraduate Award (UPA) and International Postgraduate Tuition Award (IPTA) during this study. The authors gratefully acknowledge the help of Dr. Cincotta in the final language editing of this paper.

Appendix A: The formula for calculating bearing fault frequencies [24]

1. Fault frequency of outer ring:

$$
F_{\text {OR }}=\left|\frac{\left|R_{r p m}-O R_{r p m}\right|}{2} \cdot\left[1-\frac{(\cos (\alpha)) \cdot d_{r}}{d_{m}}\right]\right| \cdot z
$$

2. Fault frequency of inner ring:

$$
F_{I R}=\left|\frac{\left|I R_{r p m}-O R_{r p m}\right|}{2} \cdot\left[1+\frac{(\cos (\alpha)) \cdot d_{r}}{d_{m}}\right]\right| \cdot z
$$

3. Fault frequency of rolling element:

$$
F_{R}=\left|\frac{\left|R_{r p m}-O R_{r p m}\right|}{2} \cdot\left[\frac{d_{m}}{d_{r}}-\frac{(\cos (\alpha))^{2} \cdot d_{r}}{d_{m}}\right]\right|
$$

where $I R_{r p m}$ and $O R_{r p m}$ are the rotational speeds of the inner ring and outer ring. For $1 \mathrm{rpm}$ the value of $I R_{r p m}$ is 1 and the value of $O R_{r p m}$ is $0 . d_{m}$ denotes the mean bearing diameter, $d_{r}$ is diameter of the rolling element and $z$ is number of rolling elements. 
Appendix B: The definition and the formula for evaluation of features extracted from laboratory slew bearing data

1. Count of high peaks $\left(E_{1}\right)$ : Since the deterioration of the slew bearing can be detected by the peaks in the monitored parameter or feature, this criterion counts the number of peaks which exceed the predetermined threshold level. The greater the number of such peaks detected, the more damaged the slew bearing. The formula for this calculation $\left(E_{1}\right)$ is as follows:

$$
E_{1}=C \times C
$$

where $C$ is the number of peaks which exceed the predetermined threshold level (threshold = $12)$, and $c$ is the constant to normalize the $E_{1}$ value $(c=0.1)$. It should be noted that each criterion value is expected to have a normalization value of less than 1 . This is illustrated below:
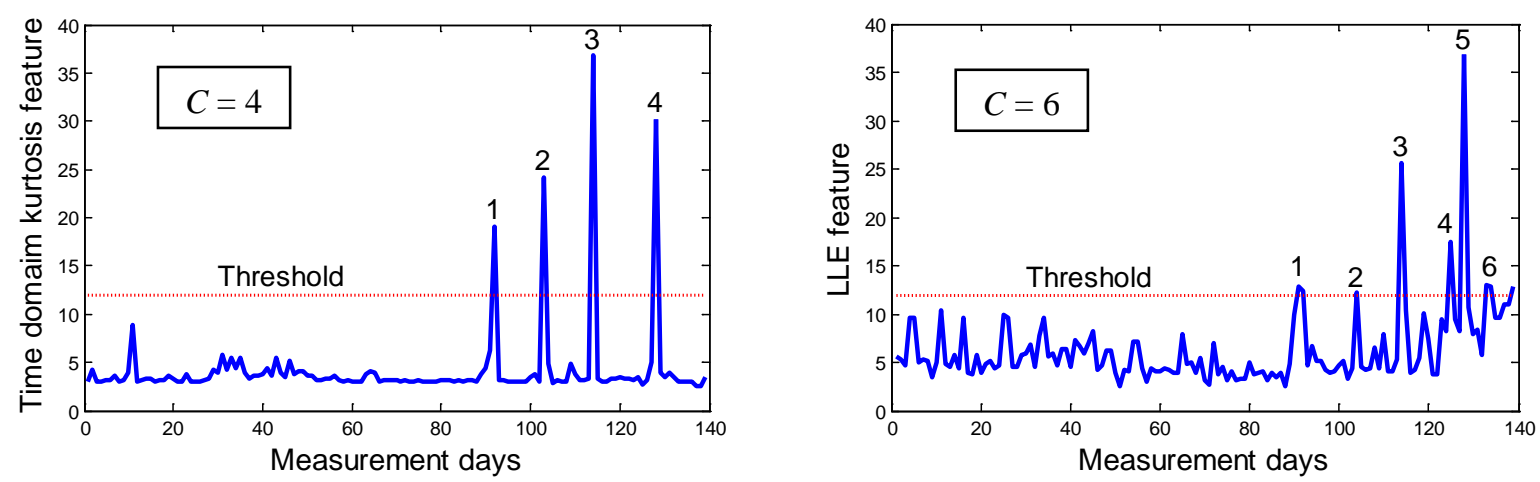

2. High peak difference $\left(E_{2}\right)$ : This criterion calculates the difference in the high peaks between anterior peaks and the detection of the first peak $\left(P_{1}\right) . E_{2}$ is given by:

$$
E_{2}=\frac{\frac{1}{C-1} \sum_{a=2}^{C}\left(P_{a}-P_{1}\right)}{\bar{P}}
$$

where $P_{a}$ is the anterior peaks and $\bar{P}$ is the average peak amplitude. If the most anterior peak levels $P_{a}$ are lower than the detection of the first peak $P_{1}$, the result will be a negative value. This is illustrated below:
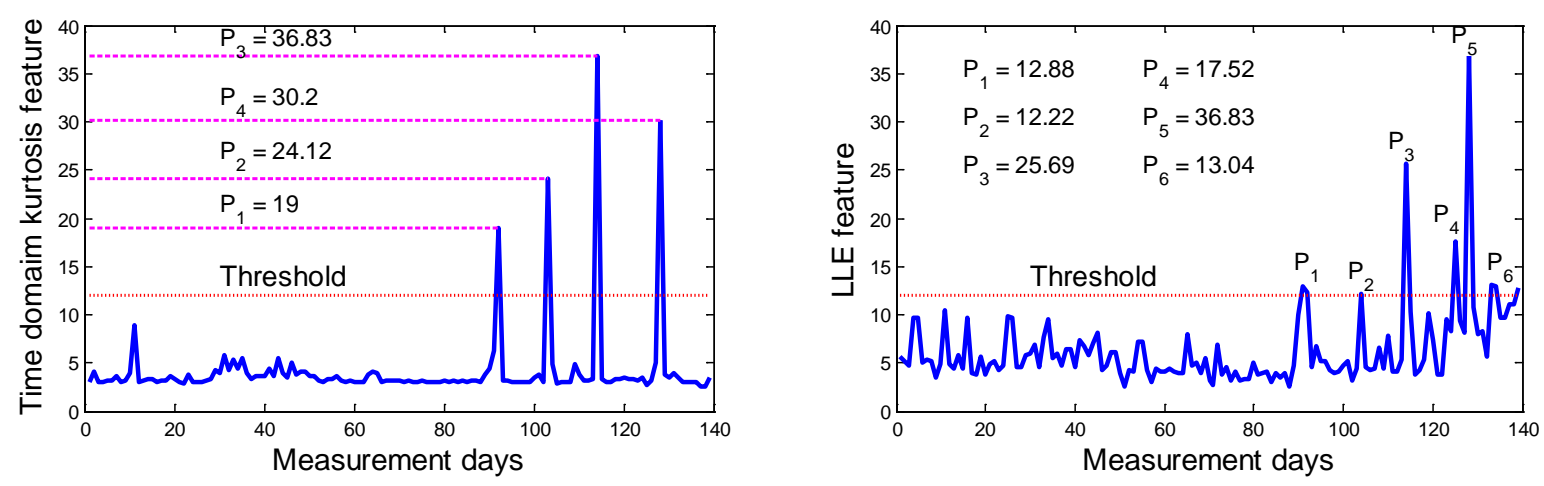
3. Time of first peak detection $\left(E_{3}\right)$ : The initial failure of the slew bearing can be identified by the day of first peak detection, according to the monitored parameter or feature. The formula of the time of first peak detection $\left(E_{3}\right)$ is given as follows:

$$
E_{3}=\left[\frac{D L-D F}{D L}\right]
$$

where $D L$ is the last measurement day (i.e.139) and $D F$ is the day of first peak detection, as shown below:
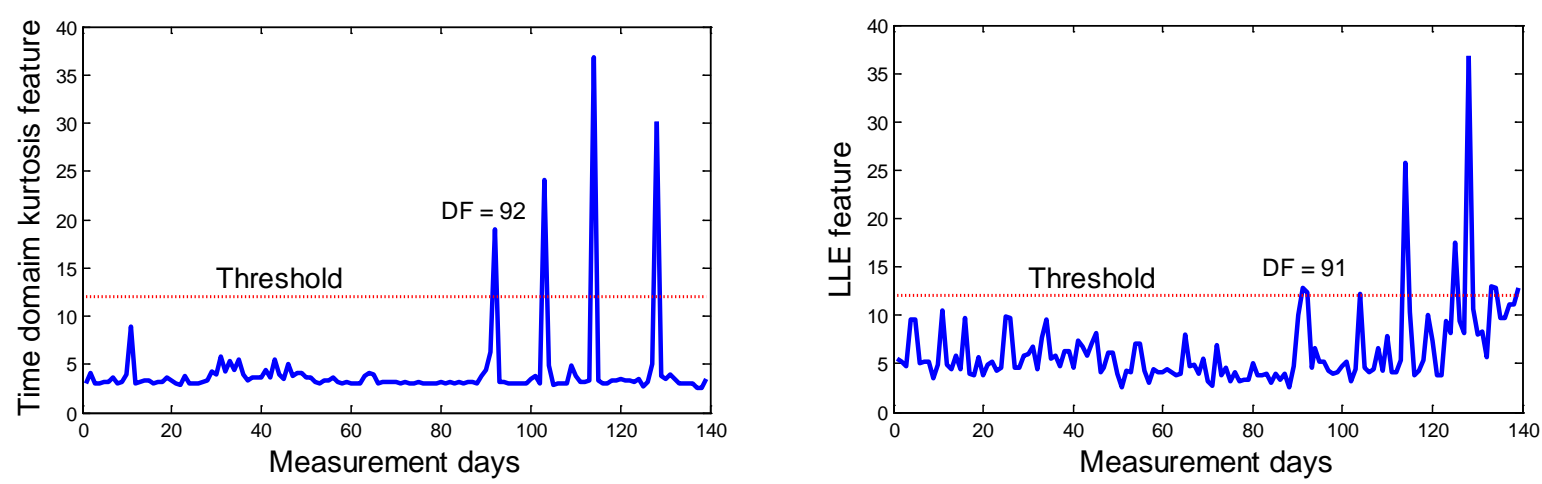

4. Interval between peaks $\left(E_{4}\right)$ : This criterion measures the interval between two adjacent peaks. If the two adjacent peaks are close, it indicates the advance of the deterioration in the condition of the slew bearing. The interval between peaks $\left(E_{4}\right)$ is calculated as follows:

$$
\begin{aligned}
& \bar{I}=\frac{\sum_{a=2}^{C}\left(D_{a}-D_{a-1}\right)}{C-1} \\
& E_{4}=\frac{D L-\bar{I}}{D L}
\end{aligned}
$$

where $\bar{I}$ is the average day interval, $D_{a}$ and $D_{a-1}$ are the anterior and the posterior day of two adjacent peaks, respectively, as shown below:
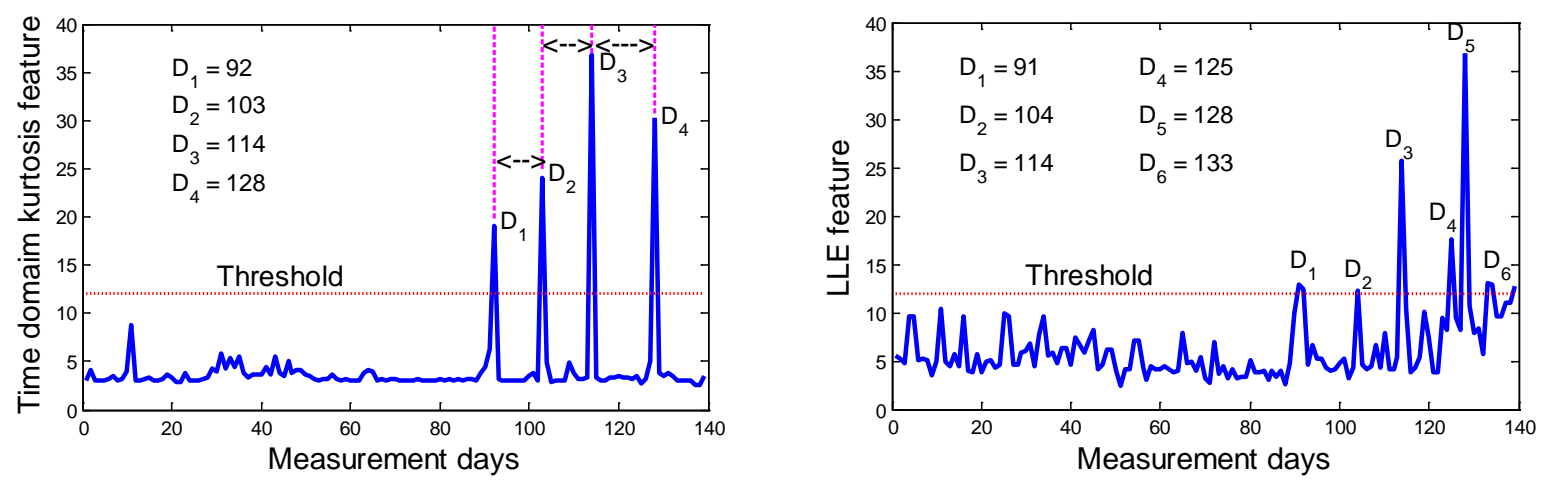
Appendix C: Illustration figures for the evaluation criteria calculation of feature extracted from coal bridge reclaimer data

1. Count of high peaks $\left(E_{1}\right)$ :
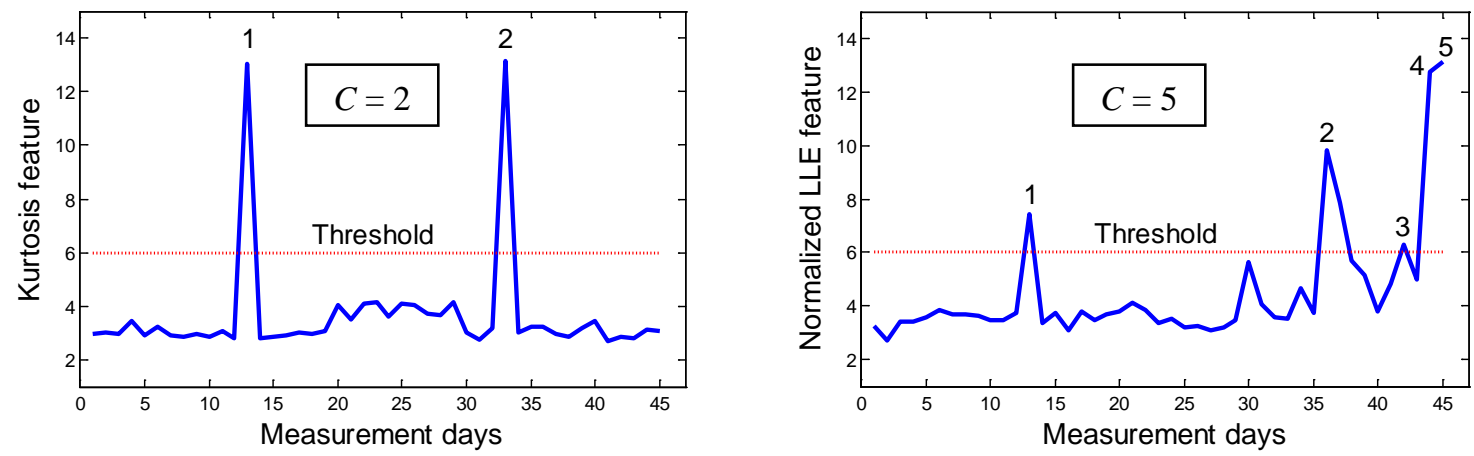

2. High peak difference $\left(E_{2}\right)$ :
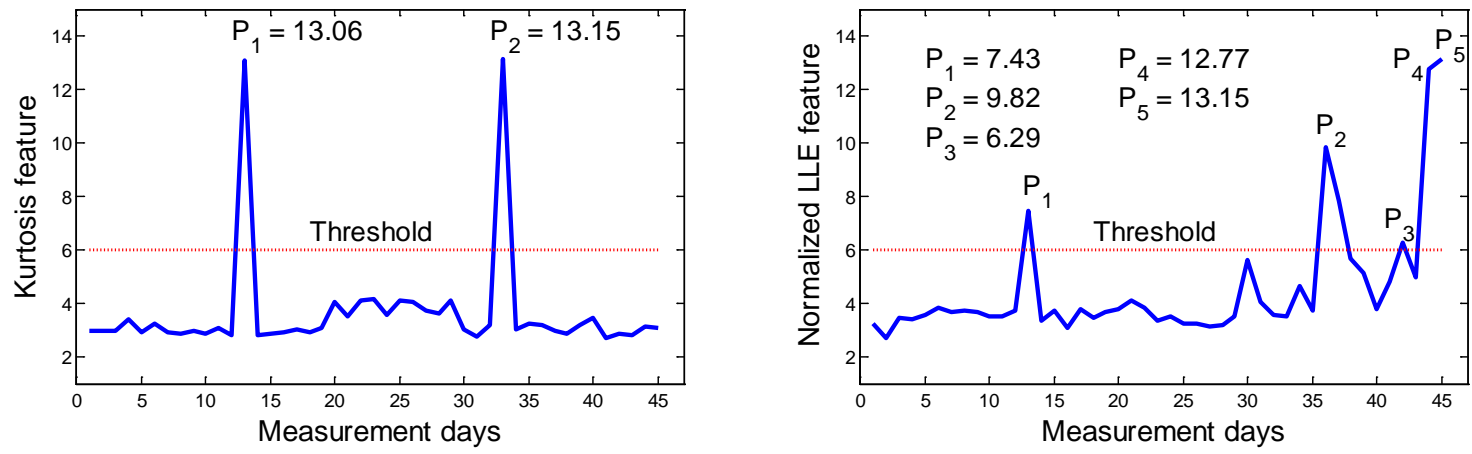

3. Time of first peak detection $\left(E_{3}\right)$ :
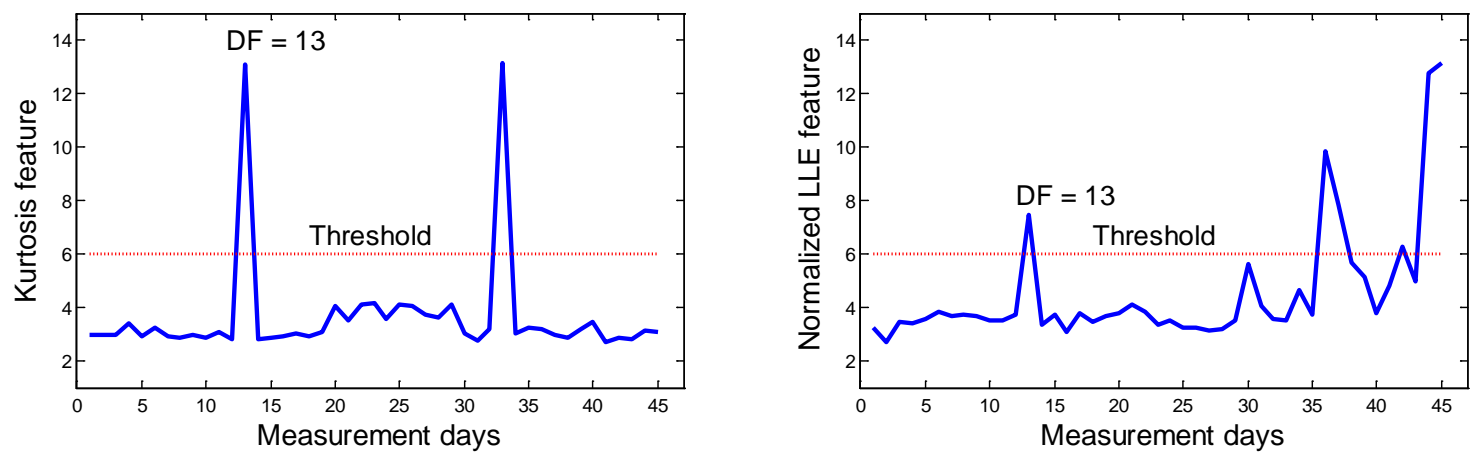

4. Interval between peaks $\left(E_{4}\right)$ :
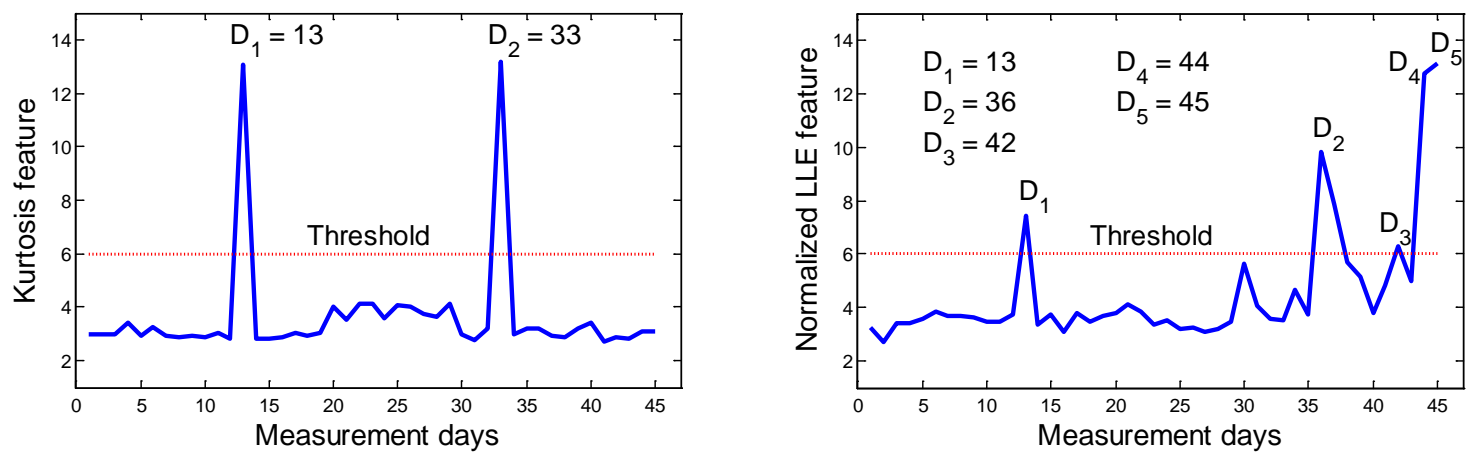


\section{References}

[1] S. Janjarasjitt, H. Ocak, K.A. Loparo, Bearing condition diagnosis and prognosis using applied nonlinear dynamical analysis of machine vibration analysis, Journal of Sound and Vibration 317 (2008) 112-126.

[2] P.F. Pai, Nonlinear vibration characterization by signal decomposition, Journal of Sound and Vibration 307 (2007) 527-544.

[3] V. Protopopescu, L.M. Hively, Phase-space dissimilarity measures of nonlinear dynamics: industrial and biomedical applications, Invited review paper in Recent Research Developments in Physics Vol. 6, Part II (2005) 649-685.

[4] G.F. Wang, Y.B. Li, Z.G. Luo, Fault classification of rolling bearing based on reconstructed phase space and Gaussian mixture model, Journal of Sound and Vibration 323 (2009) 10771089.

[5] X. Wang, V. Makis, Autoregressive model-based gear shaft fault diagnosis using the Kolmogorov-Smirnov test, Mechanical Systems and Signal Processing 327 (2009) 413-423.

[6] W. Caesarendra, B. Kosasih, K. Tieu, C.A.S. Moodie, An application of nonlinear feature extraction - A case study for low speed slewing bearing condition monitoring and prognosis, in: Proceedings of the $12^{\text {th }}$ IEEE/ASME International Conference on Advanced Intelligent Mechatronics (AIM), Wollongong, Australia, July 9-12, (2013), 1713-1718.

[7] A. Rolo-Naranjo, M.-E. Montesino-Otero, A method for the correlation dimension estimation for on-line condition monitoring of large rotating machinery, Mechanical Systems and Signal Processing 19 (2005) 939-954.

[8] Y. Ruqiang, R.X. Gao, Approximate entropy as a diagnostic tool for machine health monitoring, Mechanical Systems and Signal Processing 21 (2007) 824-839.

[9] R. Yan, Y. Liu, R.X. Gao, Permutation entropy: A nonlinear statistical measure for status characterization of rotary machines, Mechanical Systems and Signal Processing 29 (2011) 474-484.

[10] N. Paivinen, S. Lammi, A. Pitkannen, J. Nissinen, M. Penttonen, T. Gronfors, Epileptic seizure detection: A nonlinear viewpoint, Computer Methods and Programs in Biomedicine 79 (2005) 151-159.

[11] F. Shayegh, S. Sadri, R. Amirfattahi, K.A. Ansari-Asl, Model-based method for computation of correlation dimension, Lyapunov exponents and synchronization from depth-EEG signals, Computer Methods and Programs in Biomedicine 113 (2014) 323-337.

[12] M. Bask, T. Liu, A. Widerberg, The stability of electricity prices: Estimation and inference of the Lyapunov exponents, Physica A 376 (2007) 565-572.

[13] J. Wang, Q. He, Automatic fault diagnosis of rotating machines by time-scale manifold ridge analysis, Mechanical Systems and Signal Processing 40 (2013) 237-256.

[14] F. Takens, Detecting strange attractors in turbulence. in: Lecture Notes in Mathematics; Springer, New York, 1981; vol. 989, pp. 366-381. 
[15] M.T. Rosenstein, J.J. Collins, C.J. De Luca, A practical method for calculating largest Lyapunov exponents from small data sets, Physica D 65 (1993) 117-134.

[16] S. Sato, M. Sano, Y. Sawada, Practical methods of measuring the generalized dimension and the largest Lyapunov exponent in high dimensional chaotic systems, Progress of Theoretical Physics 77 (1) (1987) 1-5.

[17] B.S. Yang, A. Widodo, Introduction of Intelligent Machine Fault Diagnosis and Prognosis, Nova Science Publishers Inc., New York, 1992.

[18] W. Caesarendra, B. Kosasih, A.K. Tieu, C.A.S. Moodie, Condition monitoring of low speed slewing bearing based on largest Lyapunov exponent algorithm and circular-domain feature extractions, in: Proceeding of the $26^{\text {th }}$ International Congress of Condition Monitoring and Diagnostic Engineering Management, Finland, June 11-13, (2013), 312-319.

[19] N.E. Huang, Z. Shen, S.R. Long, M.C. Wu, H.H. Shih, Q. Zheng, N.C. Yen, C.C. Tung, H.H. Liu, The empirical mode decomposition and the Hilbert spectrum for nonlinear and non-stationary time series analysis, Proceeding of the Royal Society A: Mathematical, Physical and Engineering Sciences 454 (1998) 903-995.

[20] N.E. Huang, S.S.P. Shen, Hilber-Huang Transform and Its Application (ed.), World Scientific Publishing Co. Pte. Ltd., Singapore (2005).

[21] W. Caesarendra, P.B. Kosasih, A.K. Tieu, C.A.S. Moodie, Condition monitoring of naturally damaged slow speed slewing bearing based on ensemble empirical mode decomposition, Journal of Mechanical Science and Technology 27 (8) (2013) 2253-2262.

[22] W. Caesarendra, B. Kosasih, A.K. Tieu, C.A.S. Moodie, Circular domain features based condition monitoring for low speed slewing bearing, Mechanical Systems and Signal Processing 45 (1) (2014) 114-138.

[23] G. Rilling, P. Flandrin, P. Gonçalves, On empirical mode decomposition and its algorithms, IEEE-EURASiP Workshop on Nonlinear Signal and image Processing, NSiP-03, Grado (1), June 2003.

[24] P. Eschmann, L. Hasbargen, K. Weigand, Die Wälzlagerpraxis: Handbuch für die Berechnung und Gestaltung von Lagerungen, Publisher: R. Oldenburg, München, 1953. 